\title{
The Cauchy-Neumann and Cauchy-Robin problems for a class of hyperbolic operators with double characteristics in presence of transition
}

\author{
Annamaria Barbagallo ${ }^{1} \cdot$ Vincenzo Esposito $^{1}$
}

Received: 27 April 2020 / Revised: 27 April 2020 / Accepted: 4 September 2020 /

Published online: 14 September 2020

(c) The Author(s) 2020

\section{Abstract}

The mixed Cauchy-Neumann and Cauchy-Robin problems for a class of hyperbolic operators with double characteristics in presence of transition is investigated. Some a priori estimates in Sobolev spaces with negative indexes are proved. Subsequently, existence and uniqueness results for the mixed problems are obtained.

Keywords Cauchy-Neumann problem · Cauchy-Robin problem $\cdot$ Hyperbolic equations $\cdot$ Pseudodifferential operators

Mathematics Subject Classification 35L20 · 35B45 · 47G30

\section{Introduction}

The aim of the paper is to establish existence and uniqueness results for the CauchyNeumann and Cauchy-Robin problems associated to a class of hyperbolic operators with double characteristics in presence of transition.

Let $\Omega=] 0,+\infty\left[\times \Omega_{0}\right.$, where $\Omega_{0}$ is an open set of $\mathbb{R}^{2}$ with Lipschitz boundary. Let $x=\left(x_{0}, x^{\prime}\right)$ where $x^{\prime}=\left(x_{1}, x_{2}\right)$, let $\xi=\left(\xi_{0}, \xi^{\prime}\right)$, where $\xi^{\prime}=\left(\xi_{1}, \xi_{2}\right)$. Let $D^{\prime}=\left(\partial_{x_{1}}, \beta^{2}(x) \partial_{x_{2}}\right)$ and let $L^{\prime}=\left(\partial_{x_{1}}-a_{1}(x), \beta^{2}(x) \partial_{x_{2}}-a_{2}(x)\right)$, where $\beta(x)=$ $x_{0}-\alpha\left(x^{\prime}\right)$, being $\alpha$ a real function. Let $n=\left(n_{0}, n^{\prime}\right)$ be the external normal versor to the boundary of $\Omega$, where $n^{\prime}=\left(n_{1}, n_{2}\right)$.

Annamaria Barbagallo

annamaria.barbagallo@unina.it

Vincenzo Esposito

vincenzo.esposito7@unina.it

1 Department of Mathematics and Applications "R. Caccioppoli", University of Naples Federico II, Via Cintia - Monte S.Angelo, 80126 Naples, Italy 
The mixed problems, we will study, are introduced in the sequel. Precisely, the Cauchy-Neumann problem is

$$
\begin{cases}P u=f, & \text { in } \Omega, \\ \left.u\right|_{\Omega_{0}}=0, & \left.\frac{d u}{d n}\right|_{\Omega_{0}}=0,\left.D^{\prime} u \cdot n^{\prime}\right|_{S}=0\end{cases}
$$

and the Cauchy-Robin problem is

$$
\left\{\begin{array}{l}
P u=f, \quad \text { in } \Omega, \\
\left.u\right|_{\Omega_{0}}=0,\left.\quad \frac{d u}{d n}\right|_{\Omega_{0}}=0,\left.L^{\prime} u \cdot n^{\prime}\right|_{S}=0
\end{array}\right.
$$

where $S=] 0,+\infty\left[\times \partial \Omega_{0}\right.$ and

$$
P=D_{x_{0}}^{2}-D_{x_{1}}^{2}-\beta^{2}(x) D_{x_{2}}^{2}+\sum_{j=0}^{2} a_{j}(x) D_{x_{j}}+b(x), \quad \text { in } \Omega,
$$

with coefficients belonging in $C^{\infty}(\widetilde{\Omega})$, where $\widetilde{\Omega}=\left[0,+\infty\left[\times \widetilde{\Omega}_{0}\right.\right.$, being $\widetilde{\Omega}_{0}$ an open set containing $\Omega_{0}, \operatorname{Im} a_{2}(x)=\left(x_{0}-\alpha\left(x^{\prime}\right)\right) \tilde{a}_{2}(x)$, where $\tilde{a}_{2}(x)$ is a real function and $D_{x_{j}}=\frac{1}{i} \partial_{x_{j}}, j=0,1,2$.

For every $x^{\prime}=\left(x_{1}, x_{2}\right)$ and $\xi=\left(\xi_{0}, \xi^{\prime}\right)$,

$$
p\left(x_{0}, x^{\prime}, \xi\right)=-\xi_{0}^{2}+\xi_{1}^{2}+\left(x_{0}-\alpha\left(x^{\prime}\right)\right)^{2} \xi_{2}^{2}
$$

is the principal symbol of $P$,

$$
\Sigma=\left\{\rho=\left(x_{0}, x^{\prime}, \xi\right) \in T^{*} \Omega: \xi^{\prime} \neq 0, p(\rho)=0, \nabla p(\rho)=0\right\}
$$

is the characteristic set and

$$
F_{p}(\rho)=\frac{1}{2}\left(\begin{array}{cc}
p_{x \xi}^{\prime \prime}(\rho) & p_{\xi \xi}^{\prime \prime}(\rho) \\
-p_{x x}^{\prime \prime}(\rho) & -p_{\xi x}^{\prime \prime}(\rho)
\end{array}\right), \quad \forall \rho \in \Sigma
$$

is the fundamental matrix of $P$ at $\rho$. The spectrum of $F_{p}(\rho), \operatorname{Spec}\left(F_{p}(\rho)\right)$, is very important to analyze the well-posedness of the mixed problems associated to $P$.

Hörmander proved (see [9]):

$$
z \in \operatorname{Spec}\left(F_{p}(\rho)\right) \Leftrightarrow-z, \bar{z} \in \operatorname{Spec}\left(F_{p}(\rho)\right)
$$

We have three possible cases, see for instance [8]. There exists a positive real number $\lambda$ such that $\{-\lambda, \lambda\} \subset \operatorname{Spec}\left(F_{p}(\rho)\right)$ and $\operatorname{Spec}\left(F_{p}(\rho)\right) \backslash\{-\lambda, \lambda\} \subset i \mathbb{R}$, the operator $P$ is called effectively hyperbolic at $\rho$. We introduce

$$
\Sigma_{+}=\{\rho \in \Sigma: P \text { is effectively hyperbolic at } \rho\} .
$$


Moreover, $\operatorname{Spec}\left(F_{p}(\rho)\right) \subset i \mathbb{R}$ and in the Jordan normal form of $F(\rho)$ corresponding to the eigenvalue 0 , there are only Jordan blocks of dimension 2, namely $\operatorname{Ker} F_{p}(\rho)^{2} \cap$ $\operatorname{Im} F_{p}(\rho)^{2}=\{0\}$, the operator $P$ is called non-effectively hyperbolic of type 1 at $\rho$. We set

$$
\Sigma_{-}=\{\rho \in \Sigma: P \text { is non-effectively hyperbolic of type } 1 \text { at } \rho\} \text {. }
$$

Finally, $\operatorname{Spec}\left(F_{p}(\rho)\right) \subset i \mathbb{R}$ and in the Jordan normal form of $F(\rho)$ corresponding to the eigenvalue 0 , there is only a Jordan blocks of dimension 4 and no block of dimension 3, namely $\operatorname{Ker} F_{p}(\rho)^{2} \cap \operatorname{Im} F_{p}(\rho)^{2}$ is 2-dimensional, the operator $P$ is called non-effectively hyperbolic of type 2 at $\rho$. We denote by

$$
\Sigma_{0}=\{\rho \in \Sigma: P \text { is non-effectively hyperbolic of type } 2 \text { at } \rho\} .
$$

Obviously, it follows

$$
\Sigma=\Sigma_{-} \sqcup \Sigma_{0} \sqcup \Sigma_{+}
$$

We say that we have a transition exactly if at least two among the above sets are nonempty.

In [7] the authors study the well posedness of the Cauchy problem associated to hyperbolic operators with douple characteristics in presence of transition in the cases in which $\Sigma=\Sigma_{0} \sqcup \Sigma_{+}$or $\Sigma=\Sigma_{0} \sqcup \Sigma_{-}$. In [2], a global existence and uniqueness theorem for the Cauchy problem related to the class of hyperbolic operators with double characteristics

$$
P=D_{x_{0}}^{2}-D_{x_{1}}^{2}-\left(x_{0}+\lambda-\alpha\left(x_{1}\right)\right)^{2} D_{x_{2}}^{2}, \quad \lambda \neq 0
$$

depending on the parameter $\lambda$ in the half-space $\left.\mathbb{R}^{2} \times\right] 0,+\infty$ [ is proved. In [4], the authors consider a mixed Cauchy-Dirichlet problem associated to the previous class of operators in a particular domain of $\mathbb{R}^{3}$, instead in the class, here studied, the coeffiecient $\beta$ depends only on $x_{0}$. In [3], a priori estimates for particular test functions useful for the study of a Cauchy-Dirichlet problem are established. In this paper, unlike [7], $\Sigma=\Sigma_{-} \sqcup \Sigma_{0} \sqcup \Sigma_{+}$where all the sets $\Sigma_{-}, \Sigma_{0}$ and $\Sigma_{+}$can be nonempty on the same connected components of $\Sigma$. Moreover, unlike [5], here some priori estimates for general test functions (not particular test functions as in [5]) useful for proving the existence of solutions to the mixed Cauchy-Neumann and Cauchy-Robin problems are obtained. The class of operators (3) has both the case in which $F_{p}(\rho)$ has two distinct real eigenvalues and the case in which all the eigenvalues are purely imaginary numbers can occur (see [1,2]). Precisely, if $\left|\partial_{x_{1}} \alpha\left(x^{\prime}\right)\right|<1, \beta \equiv 0$ and $\xi_{0}=\xi_{1}=0$, then $F_{p}(\rho)$ has two distint non-zero real eigenvalues. If $\left|\partial_{x_{1}} \alpha\left(x^{\prime}\right)\right|>1, \beta \equiv 0$ and $\xi_{0}=\xi_{1}=0, F_{p}(\rho)$ has two non-zero imaginary eigenvalues. Summarizing, let $\bar{\Sigma}$ be the set of points $\rho=\left(x_{0}, x^{\prime}, \xi\right)$ of $\Sigma$ such that $\beta \equiv 0$ and $\xi_{0}=\xi_{1}=0$. It results that $\rho \in \Sigma_{+}$if $\rho \in \bar{\Sigma}$ and $\left|\partial_{x_{1}} \alpha\left(x^{\prime}\right)\right|<1, \rho \in \Sigma_{-}$if $\rho \in \bar{\Sigma}$ and $\left|\partial_{x_{1}} \alpha\left(x^{\prime}\right)\right|>1$, and $\rho \in \Sigma_{0}$ if $\rho \in \bar{\Sigma}$ and $\left|\partial_{x_{1}} \alpha\left(x^{\prime}\right)\right|=1$. In this paper, the special class of operators (3), we analyze, has transition from effectively hyperbolic to non-effectively hyperbolic. In [5] 
and [6], two classes more general of hyperbolic operators with double charateristics are investigated.

Let us introduce

$$
\begin{aligned}
\Gamma & =\{x \in \bar{\Omega}: \beta(x)=0\}, \quad \widetilde{\Gamma}=\{x \in \bar{\Omega}: \beta(x)=0\}, \\
\Gamma^{\prime} & =\left\{x \in \Gamma:\left|\partial_{x_{1}} \alpha\left(x^{\prime}\right)\right| \geq 1\right\}, \quad \widetilde{\Gamma}^{\prime}=\left\{x \in \widetilde{\Gamma}:\left|\partial_{x_{1}} \alpha\left(x^{\prime}\right)\right| \geq 1\right\}, \\
\Omega_{0}^{\prime} & =\left\{x^{\prime} \in \Omega_{0}: \alpha\left(x^{\prime}\right) \geq 0,\left|\partial_{x_{1}} \alpha\left(x^{\prime}\right)\right| \geq 1\right\}, \\
\widetilde{\Omega}_{0}^{\prime} & =\left\{x^{\prime} \in \widetilde{\Omega}_{0}: \alpha\left(x^{\prime}\right) \geq 0,\left|\partial_{x_{1}} \alpha\left(x^{\prime}\right)\right| \geq 1\right\}, \\
S & =\partial \Omega_{0} \times[0,+\infty[,
\end{aligned}
$$

furthermore $g\left(x^{\prime}\right)=\frac{\alpha\left(x^{\prime}\right)}{\partial_{x_{1}} \alpha\left(x^{\prime}\right)}, h\left(x^{\prime}\right)=1-\partial_{x_{1}} g\left(x^{\prime}\right)$, for every $x^{\prime} \in \Omega_{0}^{\prime}$.

We consider a quadratic matrix-function $B=\left(b_{h k}\right)_{h, k=0,1}$ whose elements are:

$$
\begin{aligned}
& b_{00}(x)=h\left(x^{\prime}\right)-2 \alpha\left(x^{\prime}\right)\left|\widetilde{a}_{0}(x)\right|, \quad \forall x \in \Gamma^{\prime}, \\
& b_{01}(x)=b_{10}(x)=-g\left(x^{\prime}\right) \widetilde{a}_{0}(x)-\alpha\left(x^{\prime}\right) \widetilde{a}_{1}(x), \quad \forall x \in \Gamma^{\prime}, \\
& b_{11}(x)=h\left(x^{\prime}\right)-2\left|g\left(x^{\prime}\right) \widetilde{a}_{1}(x)\right|, \quad \forall x \in \Gamma^{\prime},
\end{aligned}
$$

where $\widetilde{a}_{0}$ and $\widetilde{a}_{1}$ are the imaginary parts of $a_{0}$ and $a_{1}$, respectively.

We suppose

(i) $h\left(x^{\prime}\right) \in\left[h_{1}, h_{2}\right], \forall x^{\prime} \in \widetilde{\Omega}_{0}^{\prime}$, with $0<h_{1}<h_{2}<4$;

(ii) the matrix-function $B$ is positive definite in $\widetilde{\Gamma}^{\prime}$, namely there exists $M>0$ such that $B\left(x^{\prime}\right) \eta \cdot \eta \geq M\|\eta\|^{2}, \forall \eta=\left(\eta_{1}, \eta_{2}\right) \neq(0,0), \forall x \in \widetilde{\Gamma}^{\prime}$;

(iii) the connected components of the curve $S \cap \Gamma^{\prime}$ lie in parallel planes to $\Omega_{0}$.

We observe that if $\widetilde{a}_{0}=\widetilde{a}_{1}=0$, on $\Gamma^{\prime}$, assumption (ii) is satisfied.

The main result of the paper is the following existence and uniqueness theorem.

Theorem 1 Let $f \in H_{l o c}^{r}(\bar{\Omega})$, with $r \geq 2$. Let us suppose that assumptions (i), (ii) and (iii) hold. The Cauchy-Neumann problem (1) and Cauchy-Robin problem (2) admit a solution $u \in H_{l o c}^{r}(\bar{\Omega})$.

Example 1 Let $P=D_{x_{0}}^{2}-D_{x_{1}}^{2}-\beta^{2}(x) D_{x_{2}}^{2}+a_{0}(x) D_{x_{0}}+\beta(x)\left(a_{1}(x) D_{x_{1}}+\right.$ $\left.a_{2}(x) D_{x_{2}}\right)+b(x)$ be a hyperbolic operator in $\left.\Omega=\right] 0,+\infty\left[\times \Omega_{0}\right.$ where $\beta(x)=$ $x_{0}-\frac{x_{1}^{2}+1}{x_{2}^{2}+4}$. It results that $\partial_{x_{1}} \alpha\left(x^{\prime}\right)=\frac{2 x_{1}}{x_{2}^{2}+4}, g\left(x^{\prime}\right)=\frac{x_{1}^{2}+1}{2 x_{1}}, h\left(x^{\prime}\right)=\frac{x_{1}^{2}+1}{2 x_{1}^{2}}$, in $\Omega_{0}$. Assumption (i) is verified in $\Omega_{0}$. Assumption (ii) holds if $\operatorname{Im}\left|a_{0}(x)\right| \leq \frac{1}{2 x_{1}^{2}}$ in $\Gamma^{\prime}$. Assumption (iii) is fulfilled if $\Gamma^{\prime}$ is constituited by arcs of hyperboles of type $x_{1}^{2}+1=$ $a\left(x_{2}^{2}+4\right)(4 a>1)$. For example if $\Omega_{0}=\left\{x^{\prime} \in \mathbb{R}^{2}: x_{1}^{2}+1 \leq a\left(x_{2}^{2}+4\right), x_{2}^{2} \leq \gamma\left(x_{1}\right)\right\}$, with $a>2$ and $\gamma \in C^{1}(\mathbb{R})$ such that $\gamma\left(x_{1}\right) \geq 4(a-1)$, assumptions (i) and (iii) are satisfied and assumption (ii) is verified if $\left|\operatorname{Im} a_{0}\right|<\frac{1}{8 a^{2}}$. Furthermore we have a transition on $\Sigma$. 
Example 2 Let $P=D_{x_{0}}^{2}-D_{x_{1}}^{2}-\beta^{2}(x) D_{x_{2}}^{2}+b(x)$ be a hyperbolic operator in $\Omega=] 0,+\infty\left[\times \Omega_{0}\right.$ with $\beta(x)=x_{0}-\left(x_{1}+x_{2}\right)^{2}$. We have $\partial_{x_{1}} \alpha\left(x^{\prime}\right)=2 x_{1}, g\left(x^{\prime}\right)=$ $\frac{x_{1}^{2}+x_{2}^{2}}{2 x_{1}}, h\left(x^{\prime}\right)=\frac{x_{1}^{2}+x_{2}^{2}}{2 x_{1}}$, in $\Omega_{0}$. Assumption (i) is verified if $\left|x_{2}\right| \leq \frac{7}{4}$ and $\left|x_{1}\right| \geq \frac{1}{2}$. Assumption (ii) is always satisfied. Assumption (iii) holds if $\Gamma^{\prime}$ is constituted by arcs of circumferences with center $(0,0)$. For example if $\Omega_{0}$ is the circle in $\mathbb{R}^{2}$ with center $(0,0)$ and radius $r$ with $\frac{1}{2}<r<2$, assumptions (i), (ii) and (iii) are fulfilled and we have a transition on $\Sigma$.

The paper is organized as follows. In Sect. 2 some preliminary notations and definitions are presented. Section 3 is devoted to a priori estimates near the boundary $\Omega_{0}$. In Sect. 4 a priori estimates away from $\Omega_{0}$ are established. Section 5 concerns estimates in Sobolev spaces making use of the pseudodifferential operator theory. Section 6 deals with some global estimates in $\Omega$. In Sect. 7 existence and regularity results for solutions to the mixed Cauchy-Neumann and Cauchy-Robin problems are proved. At last, in Sect. 8, a uniqueness result for the mixed problems is obtained.

\section{Notations and preliminaries}

Let $\alpha=\left(\alpha_{0}, \alpha_{1}, \alpha_{2}\right) \in \mathbb{N}_{0}^{3}$. Let $\partial^{\alpha}$ be the derivative of order $|\alpha|$, let $\partial_{x_{j}}^{h}$ be the derivative of order $h$ with respect to $x_{j}$ and let $\partial_{x_{j}, x_{p}}^{h}$ be the derivative of order $h$ with respect to $x_{j}$ and $x_{p}$.

We indicate the $L^{2}$-scalar product, the $L^{2}$-norm and the $H^{r}$-norm by $(\cdot, \cdot),\|\cdot\|$ and $\|\cdot\|_{H^{r}}\left(r \in \mathbb{N}_{0}\right)$, respectively.

Let $C_{0}^{\infty}(\bar{\Omega})$ be the space of the restrictions to $\bar{\Omega}$ of functions belonging to $C_{0}^{\infty}\left(\mathbb{R}^{3}\right)$. For each $K \subseteq \bar{\Omega}$ compact set, let $C_{0}^{\infty}(K)$ be the set of functions $\varphi \in C_{0}^{\infty}(\bar{\Omega})$ having support contained in $K$. Set $\Omega_{k}=\left[0, k\left[\times \Omega_{0}\right.\right.$, with $k>0$, let

$$
C_{0}^{\infty}\left(\bar{\Omega}_{k}\right)=\left\{u \in C_{0}^{\infty}(\bar{\Omega}): \operatorname{supp} u \subseteq\left[0, k\left[\times \bar{\Omega}_{0}\right\}\right.\right.
$$

Let $S\left(\mathbb{R}^{3}\right)$ be the space of rapidly decreasing functions. In particular, let $S(\bar{\Omega})$ be the space of the restrictions to $\bar{\Omega}$ of functions belonging to $S\left(\mathbb{R}^{3}\right)$.

Fixed $s \in \mathbb{R}$, we consider the following norm

$$
\begin{aligned}
\|u\|_{H^{0, s}}^{2}= & \frac{1}{(2 \pi)^{2}} \int_{0}^{+\infty} d x_{0} \int_{\mathbb{R}^{2}}\left(1+\left|\xi^{\prime}\right|^{2}\right)^{s}\left|\widehat{u}\left(x_{0}, \xi^{\prime}\right)\right|^{2} d \xi^{\prime}, \\
& \forall u \in C_{0}^{\infty}(\bar{\Omega}): \operatorname{supp} u \subseteq\left[0,+\infty\left[\times \Omega_{0},\right.\right.
\end{aligned}
$$

where the Fourier transform is computed only with respect to the variable $x^{\prime}$. Let us introduce the pseudodifferential operator $A_{s}: C_{0}^{\infty}(\Omega) \rightarrow C^{\infty}(\Omega)$ given by

$$
A_{s} u=\frac{1}{(2 \pi)^{2}} \int_{\mathbb{R}^{2}} e^{i x^{\prime} \cdot \xi^{\prime}}\left(1+\left|\xi^{\prime}\right|^{2}\right)^{\frac{s}{2}} \widehat{u}\left(x_{0}, \xi^{\prime}\right) d \xi^{\prime}
$$




$$
\forall u \in C_{0}^{\infty}(\bar{\Omega}): \operatorname{supp} u \subseteq\left[0,+\infty\left[\times \Omega_{0} .\right.\right.
$$

For every $\varphi\left(x^{\prime}\right) \in C_{0}^{\infty}\left(\Omega_{0}\right)$, the operator $\varphi A_{s} u$ extends as a linear continuous operator from $H_{\text {comp. }}^{0, r}(\Omega)$ into $H_{l o c}^{0, r-s}(\Omega)$, where $r, s \in \mathbb{R}$. If supp $\varphi \subseteq \Omega_{0} \backslash \operatorname{supp} u$, then $\varphi A_{s} u$ is a regularizing operator with respect to the variable $x^{\prime}$. It results

$$
\left\|\varphi A_{s} u\right\|_{H^{0, r}} \leq c\|u\|_{H^{0, r^{\prime}}}, \quad \forall r, r^{\prime} \in \mathbb{R}, u \in C^{\infty}(\bar{\Omega}): \operatorname{supp} u \subseteq\left[0,+\infty\left[\times \Omega_{0} .\right.\right.
$$

We note that the norms $\|u\|_{H^{0, s}(\Omega)}$ and $\left\|A_{s} u\right\|_{L^{2}(\Omega)}$ are equivalent for any $s \in \mathbb{R}$. Moreover, let $H^{0, s}\left(\Omega_{k}\right)$ be the space of $u \in H^{0, s}\left(\Omega_{k}\right)$ such that supp $u \subseteq \Omega_{k}$.

Let $s \in \mathbb{R}$ and $p \geq 0$. Let $H^{p, s}\left(\mathbb{R}^{3}\right)$ be the space of all the distributions on $\mathbb{R}^{3}$ such that

$$
\|u\|_{H^{p, s}\left(\mathbb{R}^{3}\right)}^{2}=\frac{1}{(2 \pi)^{2}} \sum_{|h| \leq p} \int_{\mathbb{R}^{3}}\left(1+\left|\xi^{\prime}\right|^{2}\right)^{s}\left|\partial_{x_{0}}^{h} \widehat{u}\left(x_{0}, \xi^{\prime}\right)\right|^{2} d x_{0} d \xi^{\prime}<+\infty
$$

Let $H^{p, s}(\Omega)$ be the space of all the restrictions to $\Omega$ of elements of $H^{p, s}\left(\mathbb{R}^{3}\right)$ endowed with the norm

$$
\|u\|_{H^{p, s}(\Omega)}=\inf _{\substack{\left.U \in H^{p, s}\left(\mathbb{R}^{3}\right) \\ U\right|_{\Omega}=u}}\|U\|_{H^{p, s}\left(\mathbb{R}^{3}\right)}
$$

Analogously we can define the space $H^{p, s}\left(\Omega_{k}\right)$.

Finally, let us consider the transposed operator ${ }^{t} P$ given by:

$$
\begin{aligned}
{ }^{t} P= & -\partial_{x_{0}}^{2}+\partial_{x_{1}}^{2}+\left(x_{0}-\alpha\left(x^{\prime}\right)\right)^{2} \partial_{x_{2}}^{2}-4\left(x_{0}-\alpha\left(x^{\prime}\right)\right)\left(\partial_{x_{2}} \alpha\right) \partial_{x_{2}} \\
& -\frac{1}{i} \sum_{j=0}^{2} a_{j}(x) \partial_{x_{j}}-\frac{1}{i} \sum_{j=0}^{2} \partial_{x_{j}} a_{j}(x)-2\left(\partial_{x_{2}} \alpha\right)^{2}+b(x) .
\end{aligned}
$$

\section{A priori estimates near the boundary $\Omega_{0}$}

We enunciate the following preliminary result which synthesizes Lemmas 3.1 and 3.2 proved in [5].

Lemma 1 Let $u \in S(\bar{\Omega})$ and let $p, \alpha_{0}, \alpha_{1}, \alpha_{2} \in \mathbb{N}_{0}$. Then

$$
\left\|x_{0}^{\frac{p}{2}} \partial^{\alpha_{0}, \alpha_{1}, \alpha_{2}} u\right\| \leq \frac{2}{p+1}\left\|x_{0}^{\frac{p+2}{2}} \partial^{\alpha_{0}+1, \alpha_{1}, \alpha_{2}} u\right\|
$$

and

$$
\int_{\Omega_{0}}\left|u\left(0, x^{\prime}\right)\right|^{2} d x^{\prime} \leq 2\|u\|\left\|\partial_{x_{0}} u\right\|
$$


The proof of the following preliminary result is analogous to the one of Lemma 3.3 in [5] with some modification, therefore for reader's convenience we write it. As in Lemma 3.3 in [5], let us consider the set

$$
I_{k, \delta}=\left\{x \in \bar{\Omega}: x_{0}<k,\left|x_{0}-\alpha\left(x^{\prime}\right)\right|>\delta\right\},
$$

with $k, \delta$ positive and small enough.

Lemma 2 For every $\varepsilon, \delta>0$, there exists $k>0$ such that

$$
\begin{gathered}
\left\|\partial_{x_{0}} u\right\|+\left\|\partial_{x_{1}} u\right\|+\left\|\left(x_{0}-\alpha\left(x^{\prime}\right)\right) \partial_{x_{2}} u\right\|+\|u\| \leq \varepsilon\left\|^{t} P u\right\|, \\
\forall u \in C_{0}^{\infty}(\bar{\Omega}): \operatorname{supp} u \subseteq I_{k, \delta},\left.D^{\prime} u \cdot n^{\prime}\right|_{S}=0 .
\end{gathered}
$$

Proof Let us denote the principal part of ${ }^{t} P$ by ${ }^{t} P_{2}$, the part of the first order of ${ }^{t} P$ by ${ }^{t} P_{1}$ and the part of the zero order of ${ }^{t} P$ by ${ }^{t} P_{0}$.

Integrating by parts and taking into account the boundary conditions, we obtain

$$
\begin{aligned}
&\left(e^{\tau x_{0}} \partial_{x_{0}} u,{ }^{t} P_{2} u\right)+\left({ }^{t} P_{2} u, e^{\tau x_{0}} \partial_{x_{0}} u\right) \\
&= 2\left(e^{\tau x_{0}} \partial_{x_{0}} u(x),{ }^{t} P_{2} u\right) \\
&= \tau\left\|e^{\frac{1}{2} \tau x_{0}} \partial_{x_{0}} u\right\|^{2}+\tau\left\|e^{\frac{1}{2} \tau x_{0}} \partial_{x_{1}} u\right\|^{2}+\tau\left\|e^{\frac{1}{2} \tau x_{0}}\left(x_{0}-\alpha\left(x^{\prime}\right)\right) \partial_{x_{2}} u\right\|^{2} \\
&+2\left(e^{\tau x_{0}}\left(x_{0}-\alpha\left(x^{\prime}\right)\right) \partial_{x_{2}} u, \partial_{x_{2}} u\right) \\
&+4\left(e^{\tau x_{0}} \partial_{x_{0}} u,\left(x_{0}-\alpha\left(x^{\prime}\right)\right) \partial_{x_{2}} \alpha \partial_{x_{2}} u\right) \\
&+2 \int_{S} e^{\tau x_{0}} \partial_{x_{1}} u \cdot n_{1} \partial_{x_{0}} u d \sigma+2 \int_{S} e^{\tau x_{0}} \beta^{2}(x) \partial_{x_{2}} u \cdot n_{2} \partial_{x_{0}} u d \sigma \\
&+\int_{\Omega_{0}}\left(\partial_{x_{0}} u\right)^{2} d x^{\prime}+\int_{\Omega_{0}}\left(\partial_{x_{1}} u\right)^{2} d x^{\prime}+\int_{\Omega_{0}}\left(\left(x_{0}-\alpha\left(x^{\prime}\right)\right) \partial_{x_{2}} u\right)^{2} d x^{\prime} \\
& \geq \tau\left\|e^{\frac{1}{2} \tau x_{0}} \partial_{x_{0}} u\right\|^{2}+\tau\left\|e^{\frac{1}{2} \tau x_{0}} \partial_{x_{1}} u\right\|^{2}+\tau\left\|e^{\frac{1}{2} \tau x_{0}}\left(x_{0}-\alpha\left(x^{\prime}\right)\right) \partial_{x_{2}} u\right\|^{2} \\
&-\frac{2}{\delta}\left\|e^{\frac{1}{2} \tau x_{0}}\left(x_{0}-\alpha\left(x^{\prime}\right)\right) \partial_{x_{2}} u\right\|^{2}-2\left\|e^{\frac{1}{2} \tau x_{0}} \partial_{x_{0}} u\right\|^{2} \\
&-2\left\|e^{\frac{1}{2} \tau x_{0}}\left|\partial_{x_{2}} \alpha\left(x^{\prime}\right)\right|^{\frac{1}{2}}\left(x_{0}-\alpha\left(x^{\prime}\right)\right) \partial_{x_{2}} u\right\|^{2}, \\
& \forall u \in C_{0}^{\infty}(\bar{\Omega}): \operatorname{supp} u \subseteq I_{k, \delta},\left.D^{\prime} u \cdot n^{\prime}\right|_{S}=0 .
\end{aligned}
$$

Moreover, we have

$$
\begin{aligned}
& \left(e^{\tau x_{0}} \partial_{x_{0}} u,{ }^{t}\left(P-P_{2}\right) u\right)+\left({ }^{t}\left(P-P_{2}\right) u, e^{\tau x_{0}} \partial_{x_{0}} u\right) \\
& \quad \geq-c\left(\left\|e^{\frac{1}{2} \tau x_{0}} \partial_{x_{0}} u\right\|^{2}-\left\|e^{\frac{1}{2} \tau x_{0}} \partial_{x_{1}} u\right\|^{2}-\left\|e^{\frac{1}{2} \tau x_{0}}\left(x_{0}-\alpha\left(x^{\prime}\right)\right) \partial_{x_{2}} u\right\|^{2}\right. \\
& \left.\quad+\left\|e^{\frac{1}{2} \tau x_{0}} u\right\|\right) .
\end{aligned}
$$

Adding (5) and (6) and applying Lemma 1, it results, for $\frac{1}{2} \tau x_{0}<1$,

$$
\left(e^{\tau x_{0}} \partial_{x_{0}} u,{ }^{t} P u\right)+\left({ }^{t} P u, e^{\tau x_{0}} \partial_{x_{0}} u\right)
$$




$$
\begin{aligned}
\geq & \tau\left(\left\|\partial_{x_{0}} u\right\|^{2}+\left\|\partial_{x_{1}} u\right\|^{2}+\left\|\left(x_{0}-\alpha\left(x^{\prime}\right)\right) \partial_{x_{2}} u\right\|^{2}\right)-c\left(\frac{1}{\delta}\left\|\left(x_{0}-\alpha\left(x^{\prime}\right)\right) \partial_{x_{2}} u\right\|^{2}\right. \\
& \left.+\left\|\partial_{x_{0}} u\right\|^{2}+\left\|\partial_{x_{1}} u\right\|^{2}+\left\|\left(x_{0}-\alpha\left(x^{\prime}\right)\right) \partial_{x_{2}} u\right\|^{2}+\left\|x_{0}^{\frac{1}{2}} \partial_{x_{0}} u\right\|^{2}\right)
\end{aligned}
$$

Making use of (7) and choosing $x_{0}<\frac{1}{\tau}$, we have

$$
\begin{aligned}
&\left\|\partial_{x_{0}} u\right\|^{2}+\left\|\partial_{x_{1}} u\right\|^{2}+\left\|\left(x_{0}-\alpha\left(x^{\prime}\right)\right) \partial_{x_{2}} u\right\|^{2} \\
& \leq \frac{1}{\tau}\left\|{ }^{t} P u\right\|\left\|\partial_{x_{0}} u\right\|+\frac{c}{\tau \delta}\left\|\left(x_{0}-\alpha\left(x^{\prime}\right)\right) \partial_{x_{2}} u\right\|^{2}+\frac{c}{\tau}\left\|\partial_{x_{0}} u\right\|^{2} \\
&+\frac{c}{\tau}\left\|\left(x_{0}-\alpha\left(x^{\prime}\right)\right) \partial_{x_{2}} u\right\|^{2}+\frac{c}{\tau}\left\|\partial_{x_{1}} u\right\|^{2}+\frac{c}{\tau}\|u\|^{2} .
\end{aligned}
$$

Taking into account Lemma 1 and considering $\tau$ large enough, the claim is archieved.

Now, we establish the following result.

Theorem 2 Let us suppose that assumptions (i), (ii) and (iii) hold. Then, for every $\varepsilon>0$, there exists $k>0$ such that

$$
\begin{aligned}
& \left\|\partial_{x_{0}} u\right\|+\left\|\partial_{x_{1}} u\right\|+\left\|\left(x_{0}-\alpha\left(x^{\prime}\right)\right) \partial_{x_{2}} u\right\|+\|u\| \leq \varepsilon\left\|^{t} P u\right\|, \\
& \forall u \in C_{0}^{\infty}(\bar{\Omega}): \operatorname{supp} u \subseteq \Omega_{k}=\left[0, k\left[\times \bar{\Omega}_{0},\left.D^{\prime} u \cdot n^{\prime}\right|_{S}=0 .\right.\right.
\end{aligned}
$$

Proof If $\Gamma \cap \Omega_{0}=\emptyset$, we are able to use Lemma 2. Hence, the claim is proved. If $\Gamma \cap \Omega_{0} \neq \varnothing$, we distinguish two regions. More precisely, for every $\frac{4}{5}<\eta<1$, let

$$
\Omega_{k, \eta}=\left\{x \in \bar{\Omega}: x_{0} \in\left[0, k\left[,(1-\eta) \alpha\left(x^{\prime}\right) \leq x_{0} \leq\left(\frac{1}{5}+\eta\right) \alpha\left(x^{\prime}\right)\right\}\right.\right.
$$

and let $\Omega_{k} \backslash \Omega_{k, \eta}$. Then, let us set

$\Omega_{k, \eta}^{\prime}=\left\{x \in \Omega_{k, \eta}:\left|\partial_{x_{1}} \alpha\left(x^{\prime}\right)\right| \geq 1\right\}, \quad \Omega_{k, \eta, \eta^{\prime}}^{\prime}=\left\{x \in \Omega_{k, \eta}:\left|\partial_{x_{1}} \alpha\left(x^{\prime}\right)\right| \geq 1-\eta^{\prime}\right\}$.

Evidently $\Omega_{k, \eta, \eta^{\prime}}^{\prime} \supseteq \Omega_{k, \eta}^{\prime}$. Moreover, we choose $k, \eta$ and $\eta^{\prime}$ such that assumptions (i) and (ii) are satisfied. Let us consider a function $u \in C_{0}^{\infty}(\bar{\Omega})$ with supp $u \subseteq \Omega_{k, \eta, \eta^{\prime}}^{\prime}$ and $\left.D^{\prime} u \cdot n^{\prime}\right|_{S}=0$. Let us remark that $\Omega_{k, \eta} \cap \Omega_{0}$ has measure zero in $\mathbb{R}^{2}$. Moreover, $\Omega_{k, \eta} \cap S$ is empty or has measure zero in $\mathbb{R}^{2}$, for $k$ small enough. Let us consider the inner products:

$$
\left({ }^{t} P u, A u\right)+\left(A u,{ }^{t} P u\right), \quad \forall u \in C_{0}^{\infty}(\bar{\Omega}): \operatorname{supp} u \subseteq \Omega_{k, \eta, \eta^{\prime}}^{\prime},\left.D^{\prime} u \cdot n^{\prime}\right|_{S}=0,
$$

where $A u=x_{0} \partial_{x_{0}} u+g\left(x^{\prime}\right) \partial_{x_{1}} u$. It results

$$
\left({ }^{t} P u, A u\right)+\left(A u,{ }^{t} P u\right)=\left({ }^{t} P_{2} u, A u\right)+\left(A u,{ }^{t} P_{2} u\right)+\left({ }^{t} P_{1} u, A u\right)
$$




$$
\begin{aligned}
& +\left(A u,{ }^{t} P_{1} u\right)+\left({ }^{t} P_{0} u, A u\right)+\left(A u,{ }^{t} P_{0} u\right), \\
& \forall u \in C_{0}^{\infty}(\bar{\Omega}): \operatorname{supp} u \subseteq \Omega_{k, \eta, \eta^{\prime}}^{\prime},\left.D^{\prime} u \cdot n^{\prime}\right|_{S}=0 .
\end{aligned}
$$

Integrating by parts, for every $u \in C_{0}^{\infty}(\bar{\Omega})$ such that supp $u \subseteq \Omega_{k, \eta, \eta^{\prime}}^{\prime}$, and $\left.D^{\prime} u \cdot n^{\prime}\right|_{S}=$ 0 , we have

$$
\begin{aligned}
&\left({ }^{t} P_{2} u, A u\right)+\left(A u,{ }^{t} P_{2} u\right) \\
&= 2\left({ }^{t} P_{2} u, A u\right) \\
&= 2\left({ }^{t} P_{2} u, x_{0} \partial_{x_{0}} u\right)+2\left({ }^{t} P_{2} u, g\left(x^{\prime}\right) \partial_{x_{1}} u\right) \\
&=\left(\partial_{x_{0}} u, \partial_{x_{0}} u\right)+\left(\partial_{x_{1}} u, \partial_{x_{1}} u\right)+\left(\left(x_{0}-\alpha\left(x^{\prime}\right)\right)^{2} \partial_{x_{2}} u, \partial_{x_{2}} u\right) \\
&+2\left(\left(x_{0}-\alpha\left(x^{\prime}\right)\right) x_{0} \partial_{x_{2}} u, \partial_{x_{2}} u\right)+4\left(\left(x_{0}-\alpha\left(x^{\prime}\right)\right) \partial_{x_{2}} \alpha\left(x^{\prime}\right) \partial_{x_{2}} u, x_{0} \partial_{x_{0}} u\right) \\
&-\left(\partial_{x_{0}} u, \partial_{x_{1}} g\left(x^{\prime}\right) \partial_{x_{0}} u\right)-\left(\partial_{x_{1}} g\left(x^{\prime}\right) \partial_{x_{1}} u, \partial_{x_{1}} u\right) \\
&+4\left(\left(x_{0}-\alpha\left(x^{\prime}\right)\right) \partial_{x_{2}} \alpha\left(x^{\prime}\right) g\left(x^{\prime}\right) \partial_{x_{2}} u, \partial_{x_{1}} u\right) \\
&-2\left(\left(x_{0}-\alpha\left(x^{\prime}\right)^{2} \partial_{x_{2}} g\left(x^{\prime}\right) \partial_{x_{2}} u, \partial_{x_{1}} u\right)\right. \\
&+\left(\left(x_{0}-\alpha\left(x^{\prime}\right)\right)^{2} \partial_{x_{1}} g\left(x^{\prime}\right) \partial_{x_{2}} u, \partial_{x_{2}} u\right) \\
&-2\left(\left(x_{0}-\alpha\left(x^{\prime}\right)\right)^{2} \partial_{x_{1}} \alpha\left(x^{\prime}\right) g\left(x^{\prime}\right) \partial_{x_{2}} u, \partial_{x_{2}} u\right) .
\end{aligned}
$$

From which, it follows

$$
\begin{aligned}
& 2\left({ }^{t} P_{2} u, A u\right) \\
& =\left\|h^{\frac{1}{2}}\left(x^{\prime}\right) \partial_{x_{0}} u\right\|^{2}+\left\|h^{\frac{1}{2}}\left(x^{\prime}\right) \partial_{x_{1}} u\right\|^{2}+\left\|\left(4-h\left(x^{\prime}\right)\right)^{\frac{1}{2}}\left(x_{0}-\alpha\left(x^{\prime}\right)\right) \partial_{x_{2}} u\right\|^{2} \\
& \quad+4\left(\left(x_{0}-\alpha\left(x^{\prime}\right)\right) \partial_{x_{2}} \alpha\left(x^{\prime}\right) \partial_{x_{2}} u, x_{0} \partial_{x_{0}} u\right) \\
& \quad+4\left(\left(x_{0}-\alpha\left(x^{\prime}\right)\right) \partial_{x_{2}} \alpha\left(x^{\prime}\right) g\left(x^{\prime}\right) \partial_{x_{1}} u, \partial_{x_{2}} u\right) \\
& \quad-24\left(\left(x_{0}-\alpha\left(x^{\prime}\right)\right)^{2} \partial_{x_{2}} g\left(x^{\prime}\right) \partial_{x_{2}} u, \partial_{x_{1}} u\right)
\end{aligned}
$$

since we used that the boundary integrals are zero because the set $\Omega_{k, \eta, \eta^{\prime}}^{\prime} \cap \partial \Omega$ has zero measure.

Let us consider

$$
\begin{aligned}
& \left({ }^{t} P_{1} u, A u\right)+\left(A u,{ }^{t} P_{1} u\right) \\
& =-8\left(\left(x_{0}-\alpha\left(x^{\prime}\right)\right) \partial_{x_{2}} \alpha\left(x^{\prime}\right) \partial_{x_{2}} u, x_{0} \partial_{x_{0}} u+g\left(x^{\prime}\right) \partial_{x_{1}} u\right) \\
& \quad-2\left(\widetilde{a}_{0}(x) \partial_{x_{0}} u+\widetilde{a}_{1}(x) \partial_{x_{1}} u+\left(x_{0}-\alpha\left(x^{\prime}\right)\right) \widetilde{a}_{2}(x) \partial_{x_{2}} u, x_{0} \partial_{x_{0}} u+g\left(x^{\prime}\right) \partial_{x_{1}} u\right) \\
& =-8\left(\left(x_{0}-\alpha\left(x^{\prime}\right)\right)^{2} \partial_{x_{2}} \alpha\left(x^{\prime}\right) \partial_{x_{2}} u, \partial_{x_{0}} u\right) \\
& \quad-8\left(\left(x_{0}-\alpha\left(x^{\prime}\right)\right) \alpha\left(x^{\prime}\right) \partial_{x_{2}} \alpha\left(x^{\prime}\right) \partial_{x_{2}} u, \partial_{x_{0}} u\right) \\
& \quad-8\left(\left(x_{0}-\alpha\left(x^{\prime}\right)\right) \partial_{x_{2}} \alpha\left(x^{\prime}\right) g\left(x^{\prime}\right) \partial_{x_{2}} u, \partial_{x_{1}} u\right) \\
& \quad-2\left(\left(x_{0}-\alpha\left(x^{\prime}\right)\right) \widetilde{a}_{0}(x) \partial_{x_{0}} u, \partial_{x_{0}} u\right)
\end{aligned}
$$




$$
\begin{aligned}
& -2\left(\widetilde{a}_{0}(x) \alpha\left(x^{\prime}\right) \partial_{x_{0}} u, \partial_{x_{0}} u\right)-2\left(\widetilde{a}_{0}(x) g\left(x^{\prime}\right) \partial_{x_{0}} u, \partial_{x_{1}} u\right) \\
& -2\left(\widetilde{a}_{1}(x)\left(x_{0}-\alpha\left(x^{\prime}\right)\right) \partial_{x_{1}} u, \partial_{x_{0}} u\right)-2\left(\widetilde{a}_{1}(x) \alpha\left(x^{\prime}\right) \partial_{x_{1}} u, \partial_{x_{0}} u\right) \\
& -2\left(\widetilde{a}_{1}(x) g\left(x^{\prime}\right) \partial_{x_{1}} u, \partial_{x_{1}} u\right)-2\left(\widetilde{a}_{2}(x)\left(x_{0}-\alpha\left(x^{\prime}\right)\right)^{2} \partial_{x_{2}} u, \partial_{x_{0}} u\right) \\
& -2\left(\widetilde{a}_{2}(x)\left(x_{0}-\alpha\left(x^{\prime}\right)\right) \alpha\left(x^{\prime}\right) \partial_{x_{2}} u, \partial_{x_{0}} u\right) \\
& -2\left(\widetilde{a}_{2}(x)\left(x_{0}-\alpha\left(x^{\prime}\right)\right) g\left(x^{\prime}\right) \partial_{x_{2}} u, \partial_{x_{1}} u\right) .
\end{aligned}
$$

Making use of assumptions (i) and (ii), it follows

$$
\begin{aligned}
& \left\|h^{\frac{1}{2}}\left(x^{\prime}\right) \partial_{x_{0}} u\right\|^{2}+\left\|h^{\frac{1}{2}}\left(x^{\prime}\right) \partial_{x_{1}} u\right\|^{2}+\left\|\left(4-h\left(x^{\prime}\right)\right)^{\frac{1}{2}}\left(x_{0}-\alpha\left(x^{\prime}\right)\right) \partial_{x_{2}} u\right\|^{2} \\
& \quad-2\left(\widetilde{a}_{0}(x) \alpha\left(x^{\prime}\right) \partial_{x_{0}} u, \partial_{x_{0}} u\right)-2\left(\widetilde{a}_{0}(x) g\left(x^{\prime}\right) \partial_{x_{1}} u, \partial_{x_{0}} u\right) \\
& \quad-2\left(\widetilde{a}_{1}(x) \alpha\left(x^{\prime}\right) \partial_{x_{1}} u, \partial_{x_{0}} u\right)-2\left(\widetilde{a}_{1}(x) g\left(x^{\prime}\right) \partial_{x_{1}} u, \partial_{x_{1}} u\right) \\
& \geq L\left(\left\|\partial_{x_{0}} u\right\|^{2}+\left\|\partial_{x_{1}} u\right\|^{2}\right)+\left(4-h_{2}\right)\left\|\left(x_{0}-\alpha\left(x^{\prime}\right)\right) \partial_{x_{2}} u\right\|^{2}, \\
& \forall u \in C_{0}^{\infty}(\bar{\Omega}): \operatorname{supp} u \subseteq \Omega_{k, \eta, \eta^{\prime}}^{\prime},\left.D^{\prime} u \cdot n^{\prime}\right|_{S}=0 .
\end{aligned}
$$

Moreover, we remark that the functions $\alpha, g, \beta$ are zero on $\Omega \cap \Gamma$. As a consequence, we can choose $k$ small enough and an appropriate $\eta$ such that (12) holds and it results

$$
\begin{aligned}
& 4\left(\left(x_{0}-\alpha\left(x^{\prime}\right)\right)^{2} \partial_{x_{2}} \alpha\left(x^{\prime}\right) \partial_{x_{2}} u, \partial_{x_{0}} u\right)+4\left(\left(x_{0}-\alpha\left(x^{\prime}\right)\right) \partial_{x_{2}} \alpha\left(x^{\prime}\right) g\left(x^{\prime}\right) \partial_{x_{1}} u, \partial_{x_{2}} u\right) \\
& \quad+4\left(\left(x_{0}-\alpha\left(x^{\prime}\right)\right) \alpha\left(x^{\prime}\right) \partial_{x_{2}} \alpha\left(x^{\prime}\right) \partial_{x_{2}} u, \partial_{x_{0}} u\right) \\
& \quad-2\left(\left(x_{0}-\alpha\left(x^{\prime}\right)\right) \partial_{x_{2}} \alpha\left(x^{\prime}\right) g\left(x^{\prime}\right) \partial_{x_{2}} u, \partial_{x_{1}} u\right) \\
& \quad-2\left(\widetilde{a}_{0}(x)\left(x_{0}-\alpha\left(x^{\prime}\right)\right) \partial_{x_{0}} u, \partial_{x_{0}} u\right)-2\left(\widetilde{a}_{1}(x)\left(x_{0}-\alpha\left(x^{\prime}\right)\right) \partial_{x_{1}} u, \partial_{x_{0}} u\right) \\
& \quad-2\left(\widetilde{a}_{2}(x)\left(x_{0}-\alpha\left(x^{\prime}\right)\right)^{2} \partial_{x_{2}} u, \partial_{x_{0}} u\right)-2\left(\widetilde{a}_{2}(x)\left(x_{0}-\alpha\left(x^{\prime}\right)\right) \alpha\left(x^{\prime}\right) \partial_{x_{2}} u, \partial_{x_{0}} u\right) \\
& \quad-2\left(\widetilde{a}_{2}(x)\left(x_{0}-\alpha\left(x^{\prime}\right)\right) g\left(x^{\prime}\right) \partial_{x_{2}} u, \partial_{x_{1}} u\right) \\
& \geq-\delta\left(\left\|\partial_{x_{0}} u\right\|^{2}+\left\|\partial_{x_{1}} u\right\|^{2}+\left\|\left(x_{0}-\alpha\left(x^{\prime}\right)\right) \partial_{x_{2}} u\right\|^{2}\right),
\end{aligned}
$$

with $\delta<\min \left(L, 4-h_{2}\right)$. Adding (9), (10), (11) and taking into account (13), we have

$$
\begin{aligned}
& \left\|\partial_{x_{0}} u\right\|^{2}+\left\|\partial_{x_{1}} u\right\|^{2}+\left\|\left(x_{0}-\alpha\left(x^{\prime}\right)\right) \partial_{x_{2}} u\right\|^{2} \\
& \quad \leq c\left|\left({ }^{t} P u, A u\right)+\left(A u,{ }^{t} P u\right)\right| \\
& \quad \leq c\left(\left\|x_{0}^{\frac{1}{2} t} P u\right\|\left\|x_{0}^{\frac{1}{2}} \partial_{x_{0}} u\right\|\right. \\
& \left.\quad+\left\|g^{\frac{1}{2}}\left(x^{\prime}\right){ }^{t} P u\right\|+\left\|g^{\frac{1}{2}}\left(x^{\prime}\right) \partial_{x_{1}} u\right\|\right) .
\end{aligned}
$$

For $k$ small enough and an appropriate $\eta$, taking into account the previous inequality and Lemma 1, we obtain

$$
\begin{gathered}
\left\|\partial_{x_{0}} u\right\|+\left\|\partial_{x_{1}} u\right\|+\left\|\left(x_{0}-\alpha\left(x^{\prime}\right)\right) \partial_{x_{2}} u\right\|+\|u\| \leq \varepsilon\left\|^{t} P u\right\|, \\
\forall u \in C_{0}^{\infty}(\bar{\Omega}): \operatorname{supp} u \subseteq \Omega_{k, \eta, \eta^{\prime}}^{\prime},\left.D^{\prime} u \cdot n^{\prime}\right|_{S}=0,
\end{gathered}
$$


with $c k<\varepsilon$ and $c\left|g\left(x^{\prime}\right)\right|<\varepsilon$ in $\Omega_{k, \eta, \eta^{\prime}}^{\prime}$.

Now, we consider supp $u \subseteq \Omega_{k, \eta} \backslash \bar{\Omega}_{k, \eta, \eta^{\prime}}^{\prime}$ and we remind that it results $\left|\partial_{x_{1}} \alpha\left(x^{\prime}\right)\right|<$ 1 in $\Omega_{k, \eta} \backslash \bar{\Omega}_{k, \eta, \eta^{\prime}}^{\prime}$. Integrating by parts, we obtain

$$
\begin{aligned}
& \left({ }^{t} P u, x_{0} \partial_{x_{0}} u\right)+\left(x_{0} \partial_{x_{0}} u,{ }^{t} P u\right) \\
& =\left\|\partial_{x_{0}} u\right\|^{2}+\left\|\partial_{x_{1}} u\right\|^{2}+\left\|\left(x_{0}-\alpha\left(x^{\prime}\right)\right) \partial_{x_{2}} u\right\|^{2}+2\left(x_{0}\left(x_{0}-\alpha\left(x^{\prime}\right)\right) \partial_{x_{2}} u, \partial_{x_{2}} u\right) \\
& \quad+4\left(\left(x_{0}-\alpha\left(x^{\prime}\right)\right) \partial_{x_{2}} \alpha\left(x^{\prime}\right), x_{0} \partial_{x_{0}} u\right)+\left({ }^{t} P_{1} u, x_{0} \partial_{x_{0}} u\right)+\left(x_{0} \partial_{x_{0}} u,{ }^{t} P_{1} u\right) \\
& \quad+\left({ }^{t} P_{0} u, x_{0} \partial_{x_{0}} u\right)+\left(x_{0} \partial_{x_{0}} u,{ }^{t} P_{0} u\right), \\
& \quad \forall u \in C_{0}^{\infty}\left(\Omega_{k, \eta} \backslash \bar{\Omega}_{k, \eta, \eta^{\prime}}^{\prime}\right):\left.D^{\prime} u \cdot n^{\prime}\right|_{S}=0 .
\end{aligned}
$$

Making use of the previous inequality for $k$ small enough and Lemma 1, it follows

$$
\begin{aligned}
& \left\|\partial_{x_{0}} u\right\|^{2}+\left\|\partial_{x_{1}} u\right\|^{2}+\left\|\left(x_{0}-\alpha\left(x^{\prime}\right)\right) \partial_{x_{2}} u\right\|^{2}+\|u\|^{2} \\
& \leq \varepsilon\left\|^{t} P u\right\|^{2}+\varepsilon\left\|\left(x_{0}-\alpha\left(x^{\prime}\right)\right)^{\frac{1}{2}} \partial_{x_{2}} u\right\|^{2} .
\end{aligned}
$$

In order to estimate $\left\|\left(x_{0}-\alpha\left(x^{\prime}\right)\right)^{\frac{1}{2}} \partial_{x_{2}} u\right\|^{2}$, we consider the inner products $\left(\partial_{x_{0}} u,{ }^{t} P u\right)+$ $\left({ }^{t} P u, \partial_{x_{0}} u\right)$ and integrate by parts in the principal part for $x_{0} \leq \alpha\left(x^{\prime}\right)$ and, then, for $x_{0} \geq \alpha\left(x^{\prime}\right)$. In particular, for $x_{0} \leq \alpha\left(x^{\prime}\right)$, it results

$$
\begin{aligned}
& -\left(\left(x_{0}-\alpha\left(x^{\prime}\right)\right) \partial_{x_{2}} u, \partial_{x_{2}} u\right)+\int_{\Gamma}\left(\left(\partial_{x_{0}} u\right)^{2}+2 \partial_{x_{1}} \alpha\left(x^{\prime}\right) \partial_{x_{0}} u \partial_{x_{1}} u+\left(\partial_{x_{1}} u\right)^{2}\right) d \sigma \\
& \quad-4\left(\left(x_{0}-\alpha\left(x^{\prime}\right)\right) \partial_{x_{2}} \alpha\left(x^{\prime}\right) \partial_{x_{2}} u, \partial_{x_{0}} u\right) \\
& =-\left({ }^{t}\left(P-P_{2}\right) u, \partial_{x_{0}} u\right)-\left(\partial_{x_{0}} u,{ }^{t}\left(P-P_{2}\right) u\right) \\
& \quad+\left({ }^{t} P u, \partial_{x_{0}} u\right)+\left(\partial_{x_{0}} u,{ }^{t} P u\right), \quad \forall u \in C_{0}^{\infty}(\bar{\Omega}): \operatorname{supp} u \subseteq \Omega_{k, \eta} \backslash \Omega_{k, \eta, \eta^{\prime}}^{\prime}
\end{aligned}
$$

Moreover, for $x_{0} \geq \alpha\left(x^{\prime}\right)$, we have

$$
\begin{aligned}
& -\left(\left(x_{0}-\alpha\left(x^{\prime}\right)\right) \partial_{x_{2}} u, \partial_{x_{2}} u\right)+\int_{\Gamma}\left(\left(\partial_{x_{0}} u\right)^{2}+2 \partial_{x_{1}} \alpha\left(x^{\prime}\right) \partial_{x_{0}} u \partial_{x_{1}} u+\left(\partial_{x_{1}} u\right)^{2}\right) d \sigma \\
& \quad-4\left(\left(x_{0}-\alpha\left(x^{\prime}\right)\right) \partial_{x_{2}} \alpha\left(x^{\prime}\right) \partial_{x_{2}} u, \partial_{x_{0}} u\right) \\
& =-\left({ }^{t}\left(P-P_{2}\right) u, \partial_{x_{0}} u\right)-\left(\partial_{x_{0}} u,{ }^{t}\left(P-P_{2}\right) u\right)+\left({ }^{t} P u, \partial_{x_{0}} u\right) \\
& \quad+\left(\partial_{x_{0}} u,{ }^{t} P u\right), \quad \forall u \in C_{0}^{\infty}(\bar{\Omega}): \operatorname{supp} u \subseteq \Omega_{k, \eta} \backslash \Omega_{k, \eta, \eta^{\prime}}^{\prime}
\end{aligned}
$$

Adding (16) and (17), and taking into account that $\left|\partial_{x_{1}} \alpha\left(x^{\prime}\right)\right|<1$ in the considered part, we get

$$
\begin{aligned}
& \left\|\left(x_{0}-\alpha\left(x^{\prime}\right)\right)^{\frac{1}{2}} \partial_{x_{2}} u\right\|^{2} \\
& \quad \leq c\left(\left\|\partial_{x_{0}} u\right\|^{2}+\left\|\partial_{x_{1}} u\right\|^{2}+\left\|\left(x_{0}-\alpha\left(x^{\prime}\right)\right) \partial_{x_{2}} u\right\|^{2}+\|u\|^{2}+\left\|{ }^{t} P u\right\|^{2}\right) .
\end{aligned}
$$


Making use of (18), (15) and Lemma 1, for $k$ small enough and, hence, $\varepsilon$ small enough, it follows

$$
\begin{aligned}
& \left\|\partial_{x_{0}} u\right\|^{2}+\left\|\partial_{x_{1}} u\right\|^{2}+\left\|\left(x_{0}-\alpha\left(x^{\prime}\right)\right) \partial_{x_{2}} u\right\|^{2} \leq \varepsilon\left\|^{t} P u\right\|^{2}, \\
& \forall u \in C_{0}^{\infty}(\bar{\Omega}): \operatorname{supp} u \subseteq \Omega_{k . \eta} \backslash \Omega_{k, \eta, \eta^{\prime}}^{\prime},\left.D^{\prime} u \cdot n^{\prime}\right|_{S}=0 .
\end{aligned}
$$

Let us consider $u \in C_{0}^{\infty}(\bar{\Omega})$ such that supp $u \subseteq \Omega_{k} \backslash \bar{\Omega}_{k, \eta}$ and compute the following inner products

$$
\begin{aligned}
& \left({ }^{t} P u, x_{0} \partial_{x_{0}} u\right)+\left(x_{0} \partial_{x_{0}} u,{ }^{t} P u\right) \\
& =\left\|\partial_{x_{0}} u\right\|^{2}+\left\|\partial_{x_{1}} u\right\|^{2}+\frac{1}{2}\left\|\left(x_{0}-\alpha\left(x^{\prime}\right)\right) \partial_{x_{2}} u\right\|^{2} \\
& \quad+\left(\left(\frac{1}{2}\left(x_{0}-\alpha\left(x^{\prime}\right)\right)^{2}+2 x_{0}\left(x_{0}-\alpha\left(x^{\prime}\right)\right)\right) \partial_{x_{2}} u, \partial_{x_{2}} u\right) \\
& \quad+2 \int_{S}\left(\partial_{x_{1}} u \cdot n_{1}+\beta^{2}(x) \partial_{x_{2}} u \cdot n_{2}\right) x_{0} \partial_{x_{0}} u d \sigma \\
& \quad+4\left(x_{0}\left(x_{0}-\alpha\left(x^{\prime}\right)\right) \partial_{x_{2}} \alpha\left(x^{\prime}\right) \partial_{x_{2}} u, \partial_{x_{0}} u\right) \\
& \quad+\left({ }^{t}\left(P-P_{2}\right) u, x_{0} \partial_{x_{0}} u\right)+\left(x_{0} \partial_{x_{0}} u,{ }^{t}\left(P-P_{2}\right) u\right) \\
& \geq \\
& \quad\left\|\partial_{x_{0}} u\right\|^{2}+\left\|\partial_{x_{1}} u\right\|^{2}+\left\|\left(x_{0}-\alpha\left(x^{\prime}\right)\right) \partial_{x_{2}} u\right\|^{2} \\
& \quad-c\left(\left\|x_{0}^{\frac{1}{2}} \partial_{x_{0}} u\right\|^{2}+\left\|x_{0}^{\frac{1}{2}} \partial_{x_{1}} u\right\|^{2}+\left\|x_{0}^{\frac{1}{2}}\left(x_{0}-\alpha\left(x^{\prime}\right)\right) \partial_{x_{2}} u\right\|^{2}+\left\|x_{0}^{\frac{1}{2}} u\right\|^{2}\right) \\
& \quad+\left(\left(\frac{1}{2}\left(x_{0}-\alpha\left(x^{\prime}\right)\right)^{2}+2 x_{0}\left(x_{0}-\alpha\left(x^{\prime}\right)\right)\right) \partial_{x_{2}} u, \partial_{x_{2}} u\right) .
\end{aligned}
$$

Making use of Lemma 1 and taking $\frac{4}{5}<\eta<1$, we have $\frac{1}{2}\left(x_{0}-\alpha\left(x^{\prime}\right)\right)^{2}+$ $2 x_{0}\left(x_{0} \alpha\left(x^{\prime}\right)\right)>0$, in $\Omega_{k} \backslash \bar{\Omega}_{k, \eta}$. For $k$ small enough, it results

$$
\begin{gathered}
\left\|\partial_{x_{0}} u\right\|+\left\|\partial_{x_{1}} u\right\|+\left\|\left(x_{0}-\alpha\left(x^{\prime}\right)\right) \partial_{x_{2}} u\right\|+\|u\| \leq \varepsilon\left\|^{t} P u\right\|, \\
\forall u \in C_{0}^{\infty}(\bar{\Omega}): \operatorname{supp} u \subseteq \Omega_{k} \backslash \Omega_{k, \eta},\left.D^{\prime} u \cdot n^{\prime}\right|_{S}=0 .
\end{gathered}
$$

Since $\Omega_{0} \cap \Gamma$ has zero measure, without lost generality, we consider $u \in C_{0}^{\infty}(\bar{\Omega})$ with supp $u \subseteq \Omega_{k} \backslash\left(\Omega_{0} \cap \Gamma\right)$. Let $\varphi \in C_{0}^{\infty}(\bar{\Omega})$, with $\varphi \equiv 1$ on $\Omega_{k, \frac{4}{5}} \cap \operatorname{supp} u$, $\operatorname{supp} \varphi \subseteq \Omega_{k, \eta_{1}}$, with $\eta_{1}>\frac{4}{5}$ and $0 \leq \varphi \leq 1$ in $\bar{\Omega}$. Furthermore, let $\varphi^{\prime} \in C_{0}^{\infty}(\bar{\Omega})$, with $\varphi^{\prime} \equiv 1$ on $\Omega_{k, \eta}^{\prime}$ and $\operatorname{supp} \varphi \varphi^{\prime} \subseteq \Omega_{k, \eta_{1}, \eta^{\prime}}$. We rewrite (14) for $\varphi \varphi^{\prime} u$, with $u \in C_{0}^{\infty}(\bar{\Omega})$, and for $k$ small enough:

$$
\left\|\partial_{x_{0}} \varphi \varphi^{\prime} u\right\|+\left\|\partial_{x_{1}} \varphi \varphi^{\prime} u\right\|+\|\left(x_{0}-\alpha\left(x^{\prime}\right) \partial_{x_{2}} \varphi \varphi^{\prime} u\|\leq \varepsilon\|^{t} P \varphi \varphi^{\prime} u \| .\right.
$$

Taking into account (19), we have

$$
\begin{aligned}
& \left\|\partial_{x_{0}} \varphi\left(1-\varphi^{\prime}\right) u\right\|+\left\|\partial_{x_{1}} \varphi\left(1-\varphi^{\prime}\right) u\right\| \\
& +\|\left(x_{0}-\alpha\left(x^{\prime}\right) \partial_{x_{2}} \varphi\left(1-\varphi^{\prime}\right) u\|\leq \varepsilon\|^{t} P \varphi\left(1-\varphi^{\prime}\right) u \| .\right.
\end{aligned}
$$


Adding the previous inequalities and taking $\varepsilon$ small enough, we obtain

$$
\left\|\partial_{x_{0}} \varphi u\right\|+\left\|\partial_{x_{1}} \varphi u\right\|+\left\|\left(x_{0}-\alpha\left(x^{\prime}\right)\right) \partial_{x_{2}} \varphi u\right\|+\|\varphi u\| \leq \varepsilon\left\|^{t} P \varphi u\right\| .
$$

With analogous techniques, it follows

$$
\begin{aligned}
& \left\|\partial_{x_{0}} \varphi u\right\|+\left\|\partial_{x_{1}} \varphi u\right\|+\|\left(x_{0}-\alpha\left(x^{\prime}\right) \partial_{x_{2}} \varphi u\|+\| \varphi u \|\right. \\
& \quad \leq \varepsilon\left(\left\|\varphi^{t} P u\right\|+\left\|\left[{ }^{t} P, \varphi\right] u\right\|\right) \\
& \quad \leq \varepsilon\left\|^{t} P u\right\|+c \varepsilon\left(\left\|\partial_{x_{0}} u\right\|+\left\|\partial_{x_{1}} u\right\|+\left\|\left(x_{0}-\alpha\left(x^{\prime}\right)\right) \partial_{x_{2}} u\right\|+\|u\|\right) .
\end{aligned}
$$

Then, set $\psi=1-\varphi$, we rewrite (21) for $\psi u$

$$
\begin{aligned}
& \left\|\partial_{x_{0}} \psi u\right\|+\left\|\partial_{x_{1}} \psi u\right\|+\|\left(x_{0}-\alpha\left(x^{\prime}\right) \partial_{x_{2}} \psi u\|+\| \psi u \|\right. \\
& \quad \leq \varepsilon\left\|{ }^{t} P \psi u\right\| \\
& \quad \leq \varepsilon\left(\left\|\psi{ }^{t} P u\right\|+\left\|\left[{ }^{t} P, \psi\right] u\right\|\right) \\
& \quad \leq \varepsilon\left\|{ }^{t} P u\right\|+c \varepsilon\left(\left\|\partial_{x_{0}} u\right\|+\left\|\partial_{x_{1}} u\right\|+\left\|\left(x_{0}-\alpha\left(x^{\prime}\right)\right) \partial_{x_{2}} u\right\|+\|u\|\right) .
\end{aligned}
$$

Adding (22) and (23), it follows

$$
\begin{aligned}
& \left\|\partial_{x_{0}} u\right\|+\left\|\partial_{x_{1}} u\right\|+\|\left(x_{0}-\alpha\left(x^{\prime}\right) \partial_{x_{2}} u\|+\| u \|\right. \\
& \quad \leq \varepsilon\left\|{ }^{t} P u\right\|+c \varepsilon\left(\left\|\partial_{x_{0}} u\right\|+\left\|\partial_{x_{1}} u\right\|+\left\|\left(x_{0}-\alpha\left(x^{\prime}\right)\right) \partial_{x_{2}} u\right\|+\|u\|\right) .
\end{aligned}
$$

Then, taking $\varepsilon$ small enough, the claim is achieved.

Now, we prove the counterpart results for the Cauchy-Robin problem.

Lemma 3 For every $\varepsilon, \delta>0$ there exists $k>0$ such that

$$
\begin{gathered}
\left\|\partial_{x_{0}} u\right\|+\left\|\partial_{x_{1}} u\right\|+\left\|\left(x_{0}-\alpha\left(x^{\prime}\right)\right) \partial_{x_{2}} u\right\|+\|u\| \leq \varepsilon\left\|^{t} P u\right\|, \\
\forall u \in C_{0}^{\infty}(\bar{\Omega}): \operatorname{supp} u \subseteq I_{k, \delta},\left.L^{\prime} u \cdot n^{\prime}\right|_{S}=0 .
\end{gathered}
$$

Proof Integrating by parts, for every $u \in C_{0}^{\infty}(\bar{\Omega})$ such that supp $u \subseteq I_{k, \delta}$ and $L^{\prime} u$. $\left.n^{\prime}\right|_{S}=0$, we have

$$
\begin{aligned}
& \left({ }^{t} P_{2} u, x_{0} \partial_{x_{0}} u\right)+\left(x_{0} \partial_{x_{0}} u,{ }^{t} P_{2} u\right) \\
& =\left\|\partial_{x_{0}} u\right\|^{2}+\left\|\partial_{x_{1}} u\right\|^{2}+\left\|\left(x_{0}-\alpha\left(x^{\prime}\right)\right) \partial_{x_{2}} u\right\|^{2}+2\left(x_{0}\left(x_{0}-\alpha\left(x^{\prime}\right)\right) \partial_{x_{2}} u, \partial_{x_{2}} u\right) \\
& \quad+4\left(x_{0}\left(x_{0}-\alpha\left(x^{\prime}\right)\right) \partial_{x_{2}} \alpha\left(x^{\prime}\right) \partial_{x_{2}} u, \partial_{x_{0}} u\right) \\
& \quad+2 \int_{S}\left(\partial_{x_{1}} u \cdot n_{1}+\beta^{2}(x) \partial_{x_{2}} u \cdot n_{2}\right) x_{0} \partial_{x_{0}} u d \sigma \\
& =\left\|\partial_{x_{0}} u\right\|^{2}+\left\|\partial_{x_{1}} u\right\|^{2}+\left\|\left(x_{0}-\alpha\left(x^{\prime}\right)\right) \partial_{x_{2}} u\right\|^{2}+2\left(x_{0}\left(x_{0}-\alpha\left(x^{\prime}\right)\right) \partial_{x_{2}} u, \partial_{x_{2}} u\right) \\
& \quad+4\left(x_{0}\left(x_{0}-\alpha\left(x^{\prime}\right)\right) \partial_{x_{2}} \alpha\left(x^{\prime}\right) \partial_{x_{2}} u, \partial_{x_{0}} u\right) \\
& \quad+2 \int_{S}\left(\tilde{a}_{1}(x) n_{1}+\beta^{2}(x) \widetilde{a}_{2}(x) n_{2}\right) u x_{0} \partial_{x_{0}} u d \sigma,
\end{aligned}
$$


where we toke into account that $\left.L^{\prime} u \cdot n^{\prime}\right|_{S}=0$. It results

$$
\begin{aligned}
& \int_{S}\left(\widetilde{a}_{1}(x) n_{1}+\beta^{2}(x) \tilde{a}_{2}(x) n_{2}\right) u x_{0} \partial_{x_{0}} u d \sigma \\
& =\int_{\Omega_{k}} x_{0} \partial_{x_{1}}\left(\widetilde{a}_{1}(x) u \partial_{x_{0}} u\right) d x+\int_{\Omega_{k}} x_{0} \partial_{x_{2}}\left(\widetilde{a}_{2}(x)\left(x_{0}-\alpha\left(x^{\prime}\right)\right) u \partial_{x_{0}} u\right) d x \\
& =\frac{1}{2}\left(x_{0} \partial_{x_{1}} \widetilde{a}_{1}(x), \partial_{x_{0}} u^{2}\right)-\left(\widetilde{a}_{1}(x) u, \partial_{x_{1}} u\right)-\left(x_{0} \partial_{x_{0}} \widetilde{a}_{0}(x) u, \partial_{x_{1}} u\right) \\
& \quad+\frac{1}{2}\left(x_{0} \partial_{x_{2}}\left(\widetilde{a}_{2}(x)\left(x_{0}-\alpha\left(x^{\prime}\right)\right)\right), \partial_{x_{0}} u^{2}\right)-\left(\widetilde{a}_{2}(x)\left(x_{0}-\alpha\left(x^{\prime}\right)\right) u, \partial_{x_{2}} u\right) \\
& \quad-\left(x_{0} \partial_{x_{0}}\left(\widetilde{a}_{2}(x)\left(x_{0}-\alpha\left(x^{\prime}\right)\right)\right) u, \partial_{x_{2}} u\right) .
\end{aligned}
$$

Making use of (24), (25) and Lemma 1, it follows

$$
\begin{aligned}
& \left({ }^{t} P_{2} u, x_{0} \partial_{x_{0}} u\right)+\left(x_{0} \partial_{x_{0}} u,{ }^{t} P_{2} u\right) \\
& \geq\left\|\partial_{x_{0}} u\right\|^{2}+\left\|\partial_{x_{1}} u\right\|^{2}+\left\|\left(x_{0}-\alpha\left(x^{\prime}\right)\right) \partial_{x_{2}} u\right\|^{2}-\frac{1}{\delta}\left\|x_{0}^{\frac{1}{2}}\left(x_{0}-\alpha\left(x^{\prime}\right)\right) \partial_{x_{2}} u\right\|^{2} \\
& \quad-c\left(\left\|x_{0}^{\frac{1}{2}} \partial_{x_{0}} u\right\|^{2}-\left\|x_{0}^{\frac{1}{2}} \partial_{x_{1}} u\right\|^{2}-\left\|x_{0}^{\frac{1}{2}}\left(x_{0}-\alpha\left(x^{\prime}\right)\right) \partial_{x_{2}} u\right\|^{2}-\left\|x_{0}^{\frac{1}{2}} u\right\|^{2}\right) .
\end{aligned}
$$

On the other hand, we have

$$
\begin{aligned}
& \left({ }^{t}\left(P-P_{2}\right) u, x_{0} \partial_{x_{0}} u\right)+\left(x_{0} \partial_{x_{0}} u,{ }^{t}\left(P-P_{2}\right) u\right) \\
& \quad \geq-c\left(\left\|x_{0}^{\frac{1}{2}} \partial_{x_{0}} u\right\|^{2}-\left\|x_{0}^{\frac{1}{2}} \partial_{x_{1}} u\right\|^{2}-\left\|x_{0}^{\frac{1}{2}}\left(x_{0}-\alpha\left(x^{\prime}\right)\right) \partial_{x_{2}} u\right\|-\left\|x_{0}^{\frac{1}{2}} u\right\|^{2}\right) .
\end{aligned}
$$

Adding (26) and (27) and using Lemma 1, we obtain

$$
\begin{aligned}
& \left\|\partial_{x_{0}} u\right\|^{2}+\left\|\partial_{x_{1}} u\right\|^{2}+\left\|\left(x_{0}-\alpha\left(x^{\prime}\right)\right) \partial_{x_{2}} u\right\|^{2}+\|u\|^{2} \\
& \leq\left\|x_{0}^{\frac{1}{2}} \partial_{x_{0}} u\right\|\left\|x_{0}^{\frac{1}{2}}{ }^{t} P u\right\|+\frac{1}{\delta}\left\|x_{0}^{\frac{1}{2}}\left(x_{0}-\alpha\left(x^{\prime}\right)\right) \partial_{x_{2}} u\right\|^{2}+c\left(\left\|x_{0}^{\frac{1}{2}} \partial_{x_{0}} u\right\|^{2}\right. \\
& \left.\quad+\left\|x_{0}^{\frac{1}{2}} \partial_{x_{1}} u\right\|^{2}+\left\|x_{0}^{\frac{1}{2}}\left(x_{0}-\alpha\left(x^{\prime}\right)\right) \partial_{x_{2}} u\right\|^{2}+\left\|x_{0}^{\frac{1}{2}} u\right\|^{2}\right),
\end{aligned}
$$

from which the claim follows taking $x_{0} \leq k$ and $k$ small enough.

We prove the following result by using similar arguments as above.

Theorem 3 Let us suppose that assumptions (i), (ii) and (iii) hold. It results that for every $\varepsilon>0$ there exists $k>0$ such that

$$
\begin{aligned}
& \left\|\partial_{x_{0}} u\right\|+\left\|\partial_{x_{1}} u\right\|+\left\|\left(x_{0}-\alpha\left(x^{\prime}\right)\right) \partial_{x_{2}} u\right\|+\|u\| \leq \varepsilon\left\|^{t} P u\right\|, \\
& \forall u \in C_{0}^{\infty}(\bar{\Omega}): \operatorname{supp} u \subseteq \Omega_{k}=\left[0, k\left[\times \Omega_{0},\left.L^{\prime} u \cdot n^{\prime}\right|_{S}=0\right.\right.
\end{aligned}
$$

Proof We can proceed as in the proof of Theorem 2 making use of Lemma 3 instead of Lemma 2. Moreover, the integral $2 \int_{S}\left(\partial_{x_{1}} u \cdot n_{1}+\beta^{2}(x) \partial_{x_{2}} u \cdot n_{2}\right) x_{0} \partial_{x_{0}} u d \sigma$ in (20) 
has be estimated as in (25). More precisely, using the same arguments in (25), we obtain

$$
\begin{aligned}
& 2 \int_{S}\left(\partial_{x_{1}} u \cdot n_{1}+\beta^{2}(x) \partial_{x_{2}} u \cdot n_{2}\right) x_{0} \partial_{x_{0}} u d \sigma \\
& \quad \geq-c\left(\left\|x_{0}^{\frac{1}{2}} \partial_{x_{0}} u\right\|^{2}+\left\|x_{0}^{\frac{1}{2}} \partial_{x_{1}} u\right\|^{2}+\left\|x_{0}^{\frac{1}{2}}\left(x_{0}-\alpha\left(x^{\prime}\right)\right) \partial_{x_{2}} u\right\|^{2}-\left\|x_{0}^{\frac{1}{2}} u\right\|^{2}\right)^{2} .
\end{aligned}
$$

As a consequence, the anologous estimate of (20) can be deduced.

Taking into account Theorems 2 and 3, we deduce easily the next theorem.

Theorem 4 Let us suppose that assumptions (i), (ii) and (iii) hold. It results that for every $\varepsilon>0$ there exists $k>0$ such that

$$
\begin{gathered}
\left\|\partial_{x_{0}} u\right\|+\left\|\partial_{x_{1}} u\right\|+\left\|\left(x_{0}-\alpha\left(x^{\prime}\right)\right) \partial_{x_{2}} u\right\|+\|u\| \leq \varepsilon\left\|^{t} P u\right\|, \\
\forall u \in C_{0}^{\infty}(\overline{\widetilde{\Omega}}): \operatorname{supp} u \subseteq \widetilde{\Omega}_{k}=\left[0, k\left[\times \widetilde{\Omega}_{0} .\right.\right.
\end{gathered}
$$

\section{A priori estimates array from $\Omega_{0}$}

Let us set

$$
\begin{aligned}
& \Omega_{\bar{x}_{0}, k, \eta}=\left\{x \in \bar{\Omega}: \quad x_{0} \in\left[\bar{x}_{0}, \bar{x}_{0}+k[,\right.\right. \\
& \left.\eta \bar{x}_{0}+(1+\eta) \alpha\left(x^{\prime}\right) \leq x_{0} \leq\left(\frac{1}{5}+\eta\right) \alpha\left(x^{\prime}\right)+\left(\frac{4}{5}-\eta\right) \bar{x}_{0}\right\}, \\
& \Omega_{\bar{x}_{0}, k, \eta}^{\prime}=\left\{x \in \Omega_{\bar{x}_{0}, k, \eta}:\left|\partial_{x_{1}} \alpha\left(x^{\prime}\right)\right| \geq 1\right\}, \\
& \Omega_{\bar{x}_{0}, k, \eta, \eta^{\prime}}^{\prime}=\left\{x \in \Omega_{\bar{x}_{0}, k, \eta}:\left|\partial_{x_{1}} \alpha\left(x^{\prime}\right)\right| \geq 1-\eta^{\prime}\right\}
\end{aligned}
$$

where $\bar{x}_{0}>0, \frac{4}{5}<\eta<1, k>0$. Evidently $\Omega_{\bar{x}_{0}, k, \eta, \eta^{\prime}}^{\prime} \supseteq \Omega_{\bar{x}_{0}, k, \eta}^{\prime}$. Moreover, it is possible to choose $k, \eta, \eta^{\prime}$ such that assumptions (i) and (ii) are verified in $\Omega_{\bar{x}_{0}, k, \eta, \eta^{\prime}}^{\prime}$.

The following result holds.

Theorem 5 Let us assume that assumptions (i), (ii) and (iii) hold. Then, for every $\bar{x}_{0}>0$ and for every $\varepsilon>0$ there exists $k>0$ such that

$$
\begin{gathered}
\left\|\left(x_{0}-\alpha\left(x^{\prime}\right)\right) \partial_{x_{2}} u\right\| \leq \varepsilon\left(\left\|^{t} P u\right\|+\left\|\partial_{x_{0}} u\right\|+\left\|\partial_{x_{1}} u\right\|+\|u\|\right), \\
\forall u \in C_{0}^{\infty}(\bar{\Omega}): \operatorname{supp} u \subseteq \Omega_{\bar{x}_{0}, k, \eta},\left.D^{\prime} u \cdot n^{\prime}\right|_{S}=0 .
\end{gathered}
$$

Moreover, for every $\bar{x}_{0}>0$ there exist $k>0$ and $c>0$ such that

$$
\begin{gathered}
\left\|\partial_{x_{0}} u\right\|+\left\|\partial_{x_{1}} u\right\|+\left\|\left(x_{0}-\alpha\left(x^{\prime}\right)\right) \partial_{x_{2}} u\right\|+\|u\| \leq c\left\|^{t} P u\right\|, \\
\forall u \in C_{0}^{\infty}(\bar{\Omega}): \operatorname{supp} u \subseteq \Omega_{\bar{x}_{0}, k, \eta},\left.D^{\prime} u \cdot n^{\prime}\right|_{S}=0 .
\end{gathered}
$$


Proof If the intersection between $\Gamma$ and the plane $x_{0}=\bar{x}_{0}$ is empty, integrating by parts, as in the proof of Lemma 2, in the following inner products

$$
\left(e^{\tau\left(x_{0}-\bar{x}_{0}\right) t} P u, \partial_{x_{0}} u\right)+\left(e^{\tau\left(x_{0}-\bar{x}_{0}\right)} \partial_{x_{0}} u,{ }^{t} P u\right)
$$

we easily obtain that for every $\varepsilon>0$ there exists $k>0$ such that

$$
\begin{gathered}
\left\|\partial_{x_{0}} u\right\|+\left\|\partial_{x_{1}} u\right\|+\left\|\left(x_{0}-\alpha\left(x^{\prime}\right)\right) \partial_{x_{2}} u\right\|+\|u\| \leq \varepsilon\left\|^{t} P u\right\|, \\
\forall u \in C_{0}^{\infty}(\bar{\Omega}): \operatorname{supp} u \subseteq \Omega_{\bar{x}_{0}, k},\left.D^{\prime} u \cdot n^{\prime}\right|_{S}=0 .
\end{gathered}
$$

If the intersection between $\Gamma$ and the plane $x_{0}=\bar{x}_{0}$ is nonempty, we proceed as follows. We remark that the intersection between $\Omega_{\bar{x}_{0}, k, \eta}$ and the plane $x_{0}=\bar{x}_{0}$ has zero measure. Moreover, the intersection between $\Omega_{\bar{x}_{0}, k, \eta}$ and the surface $S$ is empty or has zero measure, for $k$ small enough. Let us set

$$
g_{\bar{x}_{0}}\left(x^{\prime}\right)=\frac{\alpha\left(x^{\prime}\right)-\bar{x}_{0}}{\partial_{x_{1}} \alpha\left(x^{\prime}\right)}, \quad h_{\bar{x}_{0}}\left(x^{\prime}\right)=\left|\frac{\partial_{x_{1}}^{2} \alpha\left(x^{\prime}\right)\left(\alpha\left(x^{\prime}\right)-\bar{x}_{0}\right)}{\left(\partial_{x_{1}} \alpha\left(x^{\prime}\right)\right)^{2}}\right| .
$$

Let us observe that, for a fixed $\varepsilon>0$, there exists $k>0$ such that $\left|x_{0}-\bar{x}_{0}\right|<\varepsilon$, $\left|\alpha\left(x^{\prime}\right)-\bar{x}_{0}\right|<\varepsilon,\left|g_{\bar{x}_{0}}\left(x^{\prime}\right)\right|<\varepsilon,\left|h_{\bar{x}_{0}}\left(x^{\prime}\right)\right|<\varepsilon$, for every $x \in \Omega_{\bar{x}_{0}, k, h}$. Let $A_{\bar{x}_{0}} u=$ $g_{\bar{x}_{0}}\left(x^{\prime}\right) \partial_{x_{1}} u+\left(x_{0}-\bar{x}_{0}\right) \partial_{x_{0}} u$. Integrating by parts in the following inner products

$$
\left({ }^{t} P u, A_{\bar{x}_{0}} u\right)+\left(A_{\bar{x}_{0}} u,{ }^{t} P u\right)
$$

and since the intersections between $\Omega \bar{x}_{0}, k, \eta$ and the plane $x_{0}=\bar{x}_{0}$ and the surface $S$, respectively, have zero measure, we have

$$
\begin{aligned}
& \left(h_{\bar{x}_{0}}\left(x^{\prime}\right) \partial_{x_{0}} u, \partial_{x_{0}} u\right)+\left(h_{\bar{x}_{0}}\left(x^{\prime}\right) \partial_{x_{1}} u, \partial_{x_{1}} u\right)+\left(\left(4-h_{\bar{x}_{0}}\left(x^{\prime}\right)\right)\left(x_{0}-\alpha\left(x^{\prime}\right)\right)^{2} \partial_{x_{2}} u, \partial_{x_{2}} u\right) \\
& \leq 4\left|\left(g_{\bar{x}_{0}}\left(x^{\prime}\right)\left(x_{0}-\alpha\left(x^{\prime}\right)\right) \partial_{x_{2}} \alpha\left(x^{\prime}\right) \partial_{x_{2}} u, \partial_{x_{1}} u\right)\right| \\
& \quad+4\left|\left(\left(x_{0}-\bar{x}_{0}\right)\left(x_{0}-\alpha\left(x^{\prime}\right)\right) \partial_{x_{2}} \alpha\left(x^{\prime}\right) \partial_{x_{2}} u, \partial_{x_{0}} u\right)\right| \\
& \quad+2\left|\left(\left(x_{0}-\alpha\left(x^{\prime}\right)\right)^{2} \partial_{x_{2}} g_{\bar{x}_{0}}\left(x^{\prime}\right) \partial_{x_{2}} u, \partial_{x_{2}} u\right)\right|+2\left|\operatorname{Re}\left({ }^{t} P_{1} u, A_{\bar{x}_{0}} u\right)\right| \\
& \quad+2\left|\operatorname{Re}\left({ }^{t} P_{0} u, A_{\bar{x}_{0}} u\right)\right|+\left|\left({ }^{t} P u, A_{\bar{x}_{0}} u\right)\right|+\left|\left(A_{\bar{x}_{0}} u,{ }^{t} P u\right)\right|, \\
& \quad \forall u \in C_{0}^{\infty}(\bar{\Omega}): \operatorname{supp} u \subseteq \Omega_{\bar{x}_{0}, k, h},\left.D^{\prime} u \cdot n^{\prime}\right|_{S}=0 .
\end{aligned}
$$

This implies

$$
\begin{gathered}
(4-\varepsilon)\left\|\left(x_{0}-\alpha\left(x^{\prime}\right)\right) \partial_{x_{2}} u\right\|^{2} \\
\leq c \varepsilon\left(\left\|\partial_{x_{0}} u\right\|^{2}+\left\|\partial_{x_{1}} u\right\|^{2}+\left\|\left(x_{0}-\alpha\left(x^{\prime}\right)\right) \partial_{x_{2}} u\right\|^{2}+\|u\|^{2}\right)+\varepsilon\left\|^{t} P u\right\|^{2}, \\
\forall u \in C_{0}^{\infty}(\bar{\Omega}): \operatorname{supp} u \subseteq \Omega_{\bar{x}_{0}, k, \eta, \eta^{\prime}},\left.D^{\prime} u \cdot n^{\prime}\right|_{S}=0 .
\end{gathered}
$$

Hence, taking $\varepsilon$ small enough, we deduce

$$
\left\|\left(x_{0}-\alpha\left(x^{\prime}\right)\right) \partial_{x_{2}} u\right\| \leq \varepsilon\left(\left\|\partial_{x_{0}} u\right\|+\left\|\partial_{x_{1}} u\right\|\right)+\varepsilon\left\|^{t} P u\right\|,
$$




$$
\forall u \in C_{0}^{\infty}(\bar{\Omega}): \operatorname{supp} u \subseteq \Omega_{\bar{x}_{0}, k, \eta, \eta^{\prime}},\left.D^{\prime} u \cdot n^{\prime}\right|_{S}=0
$$

For $\varepsilon$ small enough, it follows

$$
\begin{gathered}
\left\|\left(x_{0}-\alpha\left(x^{\prime}\right)\right) \partial_{x_{2}} u\right\| \leq \varepsilon\left(\left\|\partial_{x_{0}} u\right\|+\left\|\partial_{x_{1}} u\right\|\right)+\varepsilon\left\|^{t} P u\right\|, \\
\forall u \in C_{0}^{\infty}(\bar{\Omega}): \operatorname{supp} u \subseteq \Omega_{\bar{x}_{0}, k, \eta, \eta^{\prime}}^{\prime},\left.D^{\prime} u \cdot n^{\prime}\right|_{S}=0 .
\end{gathered}
$$

Now, we obtain (29) integrating by parts in the following inner products:

$$
\left({ }^{t} \mathrm{P} u, A u\right)+\left(A u,{ }^{t} \mathrm{P} u\right) .
$$

In fact, we have

$$
\begin{aligned}
& h_{1}\left\|\partial_{x_{0}} u\right\|^{2}+h_{1}\left\|\partial_{x_{1}} u\right\|^{2}+\left(4-h_{2}\right)\left\|\left(x_{0}-\alpha\left(x^{\prime}\right)\right) \partial_{x_{2}} u\right\|^{2} \\
& \leq c\left\|\left(x_{0}-\alpha\left(x^{\prime}\right)\right) \partial_{x_{2}} u\right\|\left(\left\|\partial_{x_{0}} u\right\|+\left\|\partial_{x_{1}} u\right\|\right)+c\left\|\left(x_{0}-\alpha\left(x^{\prime}\right)\right) \partial_{x_{0}} u\right\| \\
& \quad+\left\|\left(x_{0}-\alpha\left(x^{\prime}\right)\right) \partial_{x_{1}} u\right\|+c\left\|\left(x_{0}-\alpha\left(x^{\prime}\right)\right) \partial_{x_{1}} u\right\| \\
& \quad+c\|u\|\left(\left\|\partial_{x_{0}} u\right\|+\left\|\partial_{x_{1}} u\right\|\right)+c\left\|^{t} P u\right\|\left(\left\|\partial_{x_{0}} u\right\|+\left\|\partial_{x_{1}} u\right\|\right), \\
& \quad \forall u \in C_{0}^{\infty}(\bar{\Omega}): \operatorname{supp} u \subseteq \Omega_{\bar{x}_{0}, k, \eta, \eta^{\prime}}^{\prime}, \frac{4}{5} \leq h<1,\left.D^{\prime} u \cdot n^{\prime}\right|_{S}=0 .
\end{aligned}
$$

Making use of (32) and Lemma 1, it follows

$$
\begin{gathered}
\left\|\partial_{x_{0}} u\right\|+\left\|\partial_{x_{1}} u\right\|+\left\|\left(x_{0}-\alpha\left(x^{\prime}\right)\right) \partial_{x_{2}} u\right\|+\|u\| \leq c\left\|{ }^{t} P u\right\|, \\
\forall u \in C_{0}^{\infty}(\bar{\Omega}): \operatorname{supp} u \subseteq \Omega_{\bar{x}_{0}, k, \eta, \eta^{\prime}}^{\prime}, \frac{4}{5} \leq h<1,\left.D^{\prime} u \cdot n^{\prime}\right|_{S}=0 .
\end{gathered}
$$

If supp $u \subseteq \Omega_{\bar{x}_{0}, k, \eta} \backslash \Omega_{\bar{x}_{0}, k, \eta, \eta^{\prime}}^{\prime}$, we integrate by parts in the inner products

$$
\begin{aligned}
& \left({ }^{t} P u,\left(x_{0}-\bar{x}_{0}\right) \partial_{x_{0}} u\right)+\left(\left(x_{0}-\bar{x}_{0}\right) \partial_{x_{0}} u,{ }^{t} P u\right) \\
& \leq \\
& \quad\left\|\partial_{x_{0}} u\right\|^{2}+\left\|\partial_{x_{1}} u\right\|^{2}+\left\|\left(x_{0}-\alpha\left(x^{\prime}\right)\right) \partial_{x_{2}} u\right\|^{2} \\
& \quad+2\left(\left(x_{0}-\bar{x}_{0}\right)\left(x_{0}-\alpha\left(x^{\prime}\right)\right) \partial_{x_{2}} u, \partial_{x_{2}} u\right) \\
& \quad+4\left(\left(x_{0}-\bar{x}_{0}\right)\left(x_{0}-\alpha\left(x^{\prime}\right)\right) \partial_{x_{2}} \alpha\left(x^{\prime}\right) \partial_{x_{2}} u, \partial_{x_{0}} u\right) \\
& \quad+\left({ }^{t}\left(P-P_{2}\right) u,\left(x_{0}-\bar{x}_{0}\right) \partial_{x_{0}} u\right) \\
& \quad+\left(\left(x_{0}-\bar{x}_{0}\right) \partial_{x_{0}} u,{ }^{t}\left(P-P_{2}\right) u\right) .
\end{aligned}
$$

Then, for $k$ small enough and taking into account Lemma 1, it follows

$$
\begin{aligned}
& \left\|\partial_{x_{0}} u\right\|^{2}+\left\|\partial_{x_{1}} u\right\|^{2}+\left\|\left(x_{0}-\alpha\left(x^{\prime}\right)\right) \partial_{x_{2}} u\right\|^{2}+\|u\|^{2} \\
& \leq \varepsilon\left\|^{t} P u\right\|^{2}+\varepsilon\left(\left\|\partial_{x_{0}} u\right\|^{2}+\left\|\partial_{x_{1}} u\right\|^{2}+\left\|\left(x_{0}-\alpha\left(x^{\prime}\right)\right) \partial_{x_{2}} u\right\|^{2}+\|u\|^{2}\right) \\
& \quad+\varepsilon\left\|\left(x_{0}-\alpha\left(x^{\prime}\right)\right)^{\frac{1}{2}} \partial_{x_{2}} u\right\|^{2} .
\end{aligned}
$$


Hence, for $\varepsilon$ small enough, it results

$$
\begin{aligned}
& \left\|\partial_{x_{0}} u\right\|^{2}+\left\|\partial_{x_{1}} u\right\|^{2}+\left\|\left(x_{0}-\alpha\left(x^{\prime}\right)\right) \partial_{x_{2}} u\right\|^{2}+\|u\|^{2} \\
& \quad \leq \varepsilon\left(\left\|^{t} P u\right\|^{2}+\left\|\left(x_{0}-\alpha\left(x^{\prime}\right)\right)^{\frac{1}{2}} \partial_{x_{2}} u\right\|^{2}\right) .
\end{aligned}
$$

We estimate $\left\|\left(x_{0}-\alpha\left(x^{\prime}\right)\right) \partial_{x_{2}} u\right\|$, as done in (18). Computing the inner products

$$
\left({ }^{t} P u, \partial_{x_{0}} u\right)+\left(\partial_{x_{0}} u,{ }^{t} P u\right), \quad \forall u \in C_{0}^{\infty}(\bar{\Omega}): \operatorname{supp} u \subseteq \Omega_{\bar{x}_{0}, k, \eta} \backslash \Omega_{\bar{x}_{0}, k, \eta}^{\prime},
$$

and proceeding with the same technique, we deduce

$$
\begin{aligned}
& \left\|\left(x_{0}-\alpha\left(x^{\prime}\right)\right)^{\frac{1}{2}} \partial_{x_{2}} u\right\|^{2}+\int_{\Gamma}\left[\left(\partial_{x_{0}} u\right)^{2}+2 \partial_{x_{1}} \alpha\left(x^{\prime}\right) \partial_{x_{0}} u \partial_{x_{1}} u+\left(\partial_{x_{1}} u\right)^{2}\right] d \sigma \\
& \quad \leq\left\|{ }^{t} P u\right\|^{2}+\left|\left(\left(x_{0}-\alpha\left(x^{\prime}\right)\right) \partial_{x_{2}} u, \partial_{x_{0}} u\right)\right| .
\end{aligned}
$$

Making use of (34) and (35), we have

$$
\begin{gathered}
\left\|\partial_{x_{0}} u\right\|^{2}+\left\|\partial_{x_{1}} u\right\|^{2}+\left\|\left(x_{0}-\alpha\left(x^{\prime}\right)\right) \partial_{x_{2}} u\right\|^{2}+\|u\|^{2} \leq \varepsilon\left\|^{t} P u\right\|^{2}, \\
\forall u \in C_{0}^{\infty}(\bar{\Omega}): \operatorname{supp} u \subseteq \Omega_{\bar{x}_{0}, k, \eta} \backslash \Omega_{\bar{x}_{0}, k, \eta}^{\prime} .
\end{gathered}
$$

Since $\Gamma \cap \Omega_{\bar{x}_{0}}$ has zero measure, without lost generality, we consider $u \in C_{0}^{\infty}(\bar{\Omega})$ with supp $u \subseteq \Omega_{\bar{x}_{0}, k} \backslash\left(\Gamma \cap \Omega_{\bar{x}_{0}}\right)$, where $\Omega_{\bar{x}_{0}}=\left\{x \in \bar{\Omega}: x_{0}=\bar{x}_{0}\right\}$. Now, let $\varphi \in C_{0}^{\infty}(\bar{\Omega})$ such that $\varphi \equiv 1$ on $\Omega_{\bar{x}_{0}, k, \eta}^{\prime}$ and $\varphi \equiv 0$ on $\Omega_{\bar{x}_{0}, k, \eta} \backslash \Omega_{\bar{x}_{0}, k, \eta, \eta^{\prime}}^{\prime}$. If $u \in C_{0}^{\infty}(\bar{\Omega})$ such that supp $u \subseteq \Omega_{\bar{x}_{0}, k, \eta}$ and $\left.D^{\prime} u \cdot n^{\prime}\right|_{S}=0$, we can apply (31) to $\varphi u$ obtaining

$$
\left\|\left(x_{0}-\alpha\left(x^{\prime}\right)\right) \partial_{x_{2}} \varphi u\right\| \leq \varepsilon\left(\left\|\partial_{x_{0}} \varphi u\right\|+\left\|\partial_{x_{1}} \varphi u\right\|\right)+\varepsilon\left\|^{t} P \varphi u\right\|,
$$

then it follows

$$
\left\|\partial_{x_{0}} \varphi u\right\|+\left\|\partial_{x_{1}} \varphi u\right\|+\left\|\left(x_{0}-\alpha\left(x^{\prime}\right)\right) \partial_{x_{2}} \varphi u\right\|+\|\varphi u\| \leq c\left\|^{t} P \varphi u\right\| .
$$

On the other hand, by (36), it results

$$
\begin{aligned}
& \left\|\partial_{x_{0}}(1-\varphi) u\right\|+\left\|\partial_{x_{1}}(1-\varphi) u\right\|+\left\|\left(x_{0}-\alpha\left(x^{\prime}\right)\right) \partial_{x_{2}}(1-\varphi) u\right\| \\
& \quad+\|(1-\varphi) u\| \leq c{ }^{t} P(1-\varphi) u \| .
\end{aligned}
$$

Taking into account (37), (39) and for $\varepsilon$ small enough, we have

$$
\left\|\left(x_{0}-\alpha\left(x^{\prime}\right)\right) \partial_{x_{2}} u\right\| \leq \varepsilon\left(\left\|\partial_{x_{0}} u\right\|+\left\|\partial_{x_{1}} u\right\|+\left\|{ }^{t} P u\right\|\right) .
$$

As a consequence, (28) holds. Moreover, by (38) and (39) and for $\varepsilon$ small enough, we obtain

$$
\left\|\partial_{x_{0}} u\right\|+\left\|\partial_{x_{1}} u\right\|+\left\|\left(x_{0}-\alpha\left(x^{\prime}\right)\right) \partial_{x_{2}} u\right\|+\|u\| \leq \varepsilon\left\|^{t} P u\right\|,
$$




$$
\forall u \in C_{0}^{\infty}(\bar{\Omega}): \operatorname{supp} u \subseteq \Omega_{\bar{x}_{0}, k, \eta},\left.D^{\prime} u \cdot n^{\prime}\right|_{S}=0,
$$

and, hence, (29) is also proved.

The following results holds.

Theorem 6 For every $\bar{x}_{0}>0$ and $\varepsilon>0$ there exists $k>0$ such that for every $\eta \in] \frac{4}{5}, 1[$ it results

$$
\begin{gathered}
\left\|\partial_{x_{0}} u\right\|+\left\|\partial_{x_{1}} u\right\|+\left\|\left(x_{0}-\alpha\left(x^{\prime}\right)\right) \partial_{x_{2}} u\right\|+\|u\| \leq c\left\|{ }^{t} P u\right\|, \\
\forall u \in C_{0}^{\infty}(\bar{\Omega}): \operatorname{supp} u \subseteq \Omega_{\bar{x}_{0}, k} \backslash \Omega_{\bar{x}_{0}, k, \eta},\left.D^{\prime} u \cdot n^{\prime}\right|_{S}=0 .
\end{gathered}
$$

Proof Itegrating by parts in the following inner products

$$
\left({ }^{t} P u,\left(x_{0}-\bar{x}_{0}\right) \partial_{x_{0}} u\right)+\left(\left(x_{0}-\bar{x}_{0}\right) \partial_{x_{0}} u,{ }^{t} P u\right),
$$

we obtain

$$
\begin{aligned}
& \left\|\partial_{x_{0}} u\right\|^{2}+\left\|\partial_{x_{1}} u\right\|^{2}+\frac{1}{2}\left\|\left(x_{0}-\alpha\left(x^{\prime}\right)\right) \partial_{x_{2}} u\right\|^{2}+\frac{1}{2}\left(\left(x_{0}-\alpha\left(x^{\prime}\right)\right)^{2} \partial_{x_{2}} u, \partial_{x_{2}} u\right) \\
& \quad+2\left(\left(x_{0}-\bar{x}_{0}\right)\left(x_{0}-\alpha\left(x^{\prime}\right)\right) \partial_{x_{2}} u, \partial_{x_{2}} u\right) \\
& \leq \varepsilon\left(\left\|\partial_{x_{0}} u\right\|^{2}+\left\|\partial_{x_{1}} u\right\|^{2}+\left\|\left(x_{0}-\alpha\left(x^{\prime}\right)\right) \partial_{x_{0}} u\right\|^{2}+c\left\|\left(x_{0}-\alpha\left(x^{\prime}\right)\right) \partial_{x_{2}} u\right\|^{2}+\left\|{ }^{t} P u\right\|^{2}\right) .
\end{aligned}
$$

For $c\left|x_{0}-\bar{x}_{0}\right|<\varepsilon$, taking into account that

$$
\frac{1}{2}\left(\left(x_{0}-\alpha\left(x^{\prime}\right)\right)^{2} \partial_{x_{2}} u, \partial_{x_{2}} u\right)+2\left(\left(x_{0}-\bar{x}_{0}\right)\left(x_{0}-\alpha\left(x^{\prime}\right)\right) \partial_{x_{2}} u, \partial_{x_{2}} u\right) \geq 0,
$$

in $\Omega_{\bar{x}_{0}, k} \backslash \Omega_{\bar{x}_{0}, k, \eta}$, for $\frac{4}{5}<\eta<1$, by using Lemma 1 and for $\varepsilon$ small enough, there exists $k>0$ such that

$$
\begin{aligned}
& \left\|\partial_{x_{0}} u\right\|+\left\|\partial_{x_{1}} u\right\|+\left\|\left(x_{0}-\alpha\left(x^{\prime}\right)\right) \partial_{x_{2}} u\right\|+\|u\| \leq \varepsilon\left\|^{t} P u\right\|, \\
& \forall u \in C_{0}^{\infty}(\bar{\Omega}): \operatorname{supp} u \subseteq \Omega_{\bar{x}_{0}, k} \backslash \Omega_{\bar{x}_{0}, k, \eta, \eta^{\prime}}^{\prime},\left.D^{\prime} u \cdot n^{\prime}\right|_{S}=0 .
\end{aligned}
$$

As a consequence, the claim is achieved.

Hence, we obtain the following theorem.

Theorem 7 Let us suppose that assumptions (i), (ii) and (iii) hold. Then, for every $\bar{x}_{0}>0$ and for every $\varepsilon>0$ there exists $k>0$ such that

$$
\begin{gathered}
\left\|\left(x_{0}-\alpha\left(x^{\prime}\right)\right) \partial_{x_{2}} u\right\| \leq \varepsilon\left(\left\|{ }^{t} P u\right\|+\left\|\partial_{x_{0}} u\right\|+\left\|\partial_{x_{1}} u\right\|+\|u\|\right), \\
\forall u \in C_{0}^{\infty}(\bar{\Omega}): \operatorname{supp} u \subseteq \Omega_{\bar{x}_{0}, k},\left.D^{\prime} u \cdot n^{\prime}\right|_{S}=0 .
\end{gathered}
$$

Moreover, for every $\bar{x}_{0}>0$ there exist $k>0$ and $c>0$ such that

$$
\begin{gathered}
\left\|\partial_{x_{0}} u\right\|+\left\|\partial_{x_{1}} u\right\|+\left\|\left(x_{0}-\alpha\left(x^{\prime}\right)\right) \partial_{x_{2}} u\right\|+\|u\| \leq c\left\|^{t} P u\right\|, \\
\forall u \in C_{0}^{\infty}(\bar{\Omega}): \operatorname{supp} u \subseteq \Omega_{\bar{x}_{0}, k},\left.D^{\prime} u \cdot n^{\prime}\right|_{S}=0 .
\end{gathered}
$$


Proof Let $\varphi \in C_{0}^{\infty}(\bar{\Omega})$ such that $\varphi \equiv 1$ on $\Omega_{\bar{x}_{0}, k, \eta_{1}} \cap \operatorname{supp} u$, and let $\psi \in C_{0}^{\infty}(\bar{\Omega})$ such that $\psi \equiv 1$ on $\Omega_{\bar{x}_{0}, k} \backslash \Omega_{\bar{x}_{0}, k, \eta_{1}}$ and $\psi \equiv 0$ on $\Omega_{\bar{x}_{0}, k} \backslash \Omega_{\bar{x}_{0}, k, \eta_{2}}$, with $\frac{4}{5}<\eta_{1}<\eta_{2}<1$.

Applying Theorem 5 to $\varphi u$, Theorem 6 to $\psi u$ and adding the obtained inequalities, the claims are achieved

With analogous proof of Theorem 5, we are able to establish the following result.

Theorem 8 Let us suppose that assumptions (i), (ii) and (iii) hold. It results that for every $\bar{x}_{0}>0$ and for every $\varepsilon>0$ there exists $k>0$ such that

$$
\begin{gathered}
\left\|\left(x_{0}-\alpha\left(x^{\prime}\right)\right) \partial_{x_{2}} u\right\| \leq \varepsilon\left(\left\|{ }^{t} P u\right\|+\left\|\partial_{x_{0}} u\right\|+\left\|\partial_{x_{1}} u\right\|+\|u\|\right), \\
\forall u \in C_{0}^{\infty}(\bar{\Omega}): \operatorname{supp} u \subseteq \Omega_{\bar{x}_{0}, k, \eta},\left.L^{\prime} u \cdot n^{\prime}\right|_{S}=0 .
\end{gathered}
$$

Moreover, for every $\bar{x}_{0}>0$ there exist $k>0$ and $c>0$ such that

$$
\begin{gathered}
\left\|\partial_{x_{0}} u\right\|+\left\|\partial_{x_{1}} u\right\|+\left\|\left(x_{0}-\alpha\left(x^{\prime}\right)\right) \partial_{x_{2}} u\right\|+\|u\| \leq c\left\|^{t} P u\right\|, \\
\forall u \in C_{0}^{\infty}(\bar{\Omega}): \operatorname{supp} u \subseteq \Omega_{\bar{x}_{0}, k, \eta},\left.L^{\prime} u \cdot n^{\prime}\right|_{S}=0 .
\end{gathered}
$$

Now, we prove a useful estimate.

Theorem 9 For every $\bar{x}_{0}>0$ and $\varepsilon>0$ there exists $k>0$ such that, for every $\eta \in] \frac{4}{5}, 1[$, it results

$$
\begin{aligned}
& \left\|\partial_{x_{0}} u\right\|+\left\|\partial_{x_{1}} u\right\|+\left\|\left(x_{0}-\alpha\left(x^{\prime}\right)\right) \partial_{x_{2}} u\right\|+\|u\| \leq c\left\|^{t} P u\right\|, \\
& \forall u \in C_{0}^{\infty}\left(\Omega_{\bar{x}_{0}, k}\right): \operatorname{supp} u \subseteq \Omega_{\bar{x}_{0}, k} \backslash \Omega_{\bar{x}_{0}, k, \eta},\left.L^{\prime} u \cdot n^{\prime}\right|_{S}=0 .
\end{aligned}
$$

Proof Integrating by parts, we obtain

$$
\begin{aligned}
& \left({ }^{t} P u,\left(x_{0}-\bar{x}_{0}\right) \partial_{x_{0}} u\right)+\left(\left(x_{0}-\bar{x}_{0}\right) \partial_{x_{0}} u,{ }^{t} P u\right) \\
& =\quad\left\|\partial_{x_{0}} u\right\|^{2}+\frac{1}{2}\left\|\partial_{x_{1}} u\right\|^{2}+\frac{1}{2}\left\|\left(x_{0}-\alpha\left(x^{\prime}\right)\right) \partial_{x_{2}} u\right\|^{2} \\
& \quad+\frac{1}{2}\left(\left(x_{0}-\alpha\left(x^{\prime}\right)\right)^{2} \partial_{x_{2}} u, \partial_{x_{2}} u\right) \\
& \quad+2\left(\left(x_{0}-\bar{x}_{0}\right)\left(x_{0}-\alpha\left(x^{\prime}\right)\right) \partial_{x_{2}} u, \partial_{x_{2}} u\right) \\
& \quad+4\left(\left(x_{0}-\bar{x}_{0}\right)\left(x_{0}-\alpha\left(x^{\prime}\right)\right) \partial_{x_{2}} \alpha\left(x^{\prime}\right) \partial_{x_{2}} u, \partial_{x_{0}} u\right) \\
& \quad+2 \int_{S}\left(\partial_{x_{1}} u \cdot n_{1}+\beta^{2}(x) \partial_{x_{2}} u \cdot n_{2}\right)\left(x_{0}-\bar{x}_{0}\right) \partial_{x_{0}} u d \sigma .
\end{aligned}
$$

On the other hand, as done in (25), we have

$$
\begin{aligned}
& \int_{S}\left(\widetilde{a}_{1}(x) n_{1}+\beta(x) \tilde{a}_{2}(x) n_{2}\right) u x_{0} \partial_{x_{0}} u d \sigma \\
& =\frac{1}{2}\left(x_{0} \partial_{x_{1}} \widetilde{a}_{1}(x), \partial_{x_{0}} u^{2}\right)-\left(\widetilde{a}_{1}(x) u, \partial_{x_{1}} u\right)-\left(x_{0} \partial_{x_{0}} \widetilde{a}_{1}(x), \partial_{x_{1}} u\right) \\
& \quad+\frac{1}{2}\left(x_{0} \partial_{x_{2}}\left(\left(x_{0}-\alpha\left(x^{\prime}\right)\right) \tilde{a}_{2}(x)\right), \partial_{x_{0}} u^{2}\right)-\left(\left(x_{0}-\alpha\left(x^{\prime}\right)\right) \widetilde{a}_{2}(x) u, \partial_{x_{2}} u\right)
\end{aligned}
$$




$$
-\left(x_{0} \partial_{x_{0}}\left(\left(x_{0}-\alpha\left(x^{\prime}\right)\right) \tilde{a}_{2}(x)\right) u, \partial_{x_{2}} u\right) .
$$

Making use of (43) and (44) and taking into acount

$$
\frac{1}{2}\left(\left(x_{0}-\alpha\left(x^{\prime}\right)\right)^{2} \partial_{x_{2}} u, \partial_{x_{2}} u\right)+2\left(\left(x_{0}-\bar{x}_{0}\right)\left(x_{0}-\alpha\left(x^{\prime}\right)\right) \partial_{x_{2}} u, \partial_{x_{2}} u\right) \geq 0,
$$

for $\eta \in] \frac{4}{5}, 1[$, we deduce

$$
\begin{aligned}
& \left\|\partial_{x_{0}} u\right\|^{2}+\left\|\partial_{x_{1}} u\right\|^{2}+\left\|\left(x_{0}-\alpha\left(x^{\prime}\right)\right) \partial_{x_{2}} u\right\|^{2} \\
& \leq\left(\left\|\left(x_{0}-\bar{x}_{0}\right)^{\frac{1}{2}} \partial_{x_{0}} u\right\|^{2}+\left\|\left(x_{0}-\bar{x}_{0}\right)^{\frac{1}{2}} \partial_{x_{1}} u\right\|^{2}+\left\|\left(x_{0}-\bar{x}_{0}\right)^{\frac{1}{2}}\left(x_{0}-\alpha\left(x^{\prime}\right)\right) \partial_{x_{2}} u\right\|^{2}\right. \\
& \left.\quad+\left\|\left(x_{0}-\bar{x}_{0}\right)^{\frac{1}{2}} u\right\|^{2}+\left\|\left(x_{0}-\bar{x}_{0}\right)^{\frac{1}{2} t} P u\right\|^{2}\right) .
\end{aligned}
$$

Then, by using Lemma 1 , the claim follows taking $\left|x_{0}-\bar{x}_{0}\right|>k$, with $k$ small enough.

Proceeding as in the proof of Theorem 7, we obtain the following theorem.

Theorem 10 Let us suppose that assumptions (i), (ii) and (iii) hold. Then, for every $\bar{x}_{0}>0$ and for every $\varepsilon>0$ there exists $k>0$ such that

$$
\begin{gathered}
\left\|\left(x_{0}-\alpha\left(x^{\prime}\right)\right) \partial_{x_{2}} u\right\| \leq \varepsilon\left(\left\|^{t} P u\right\|+\left\|\partial_{x_{0}} u\right\|+\left\|\partial_{x_{1}} u\right\|+\|u\|\right), \\
\forall u \in C_{0}^{\infty}(\bar{\Omega}): \operatorname{supp} u \subseteq \Omega_{\bar{x}_{0}, k},\left.L^{\prime} u \cdot n^{\prime}\right|_{S}=0 .
\end{gathered}
$$

Moreover, for every $\bar{x}_{0}>0$ there exist $k>0$ and $c>0$ such that

$$
\begin{gathered}
\left\|\partial_{x_{0}} u\right\|+\left\|\partial_{x_{1}} u\right\|+\left\|\left(x_{0}-\alpha\left(x^{\prime}\right)\right) \partial_{x_{2}} u\right\|+\|u\| \leq c\left\|^{t} P u\right\|, \\
\forall u \in C_{0}^{\infty}(\bar{\Omega}): \operatorname{supp} u \subseteq \Omega_{\bar{x}_{0}, k},\left.L^{\prime} u \cdot n^{\prime}\right|_{S}=0 .
\end{gathered}
$$

As a consequence, the next result holds.

Theorem 11 Let us suppose that assumptions (i), (ii) and (iii) hold. Then, for every $\bar{x}_{0}>0$ and for every $\varepsilon>0$ there exists $k>0$ such that

$$
\begin{gathered}
\left\|\left(x_{0}-\alpha\left(x^{\prime}\right)\right) \partial_{x_{2}} u\right\| \leq \varepsilon\left(\left\|{ }^{t} P u\right\|+\left\|\partial_{x_{0}} u\right\|+\left\|\partial_{x_{1}} u\right\|+\|u\|\right), \\
\forall u \in C_{0}^{\infty}(\overline{\widetilde{\Omega}}): \operatorname{supp} u \subseteq \widetilde{\Omega}_{\bar{x}_{0}, k}=\left[\bar{x}_{0}, \bar{x}_{0}+k[\times \widetilde{\Omega} .\right.
\end{gathered}
$$

Moreover, for every $\bar{x}_{0}>0$ there exist $k>0$ and $c>0$ such that

$$
\begin{gathered}
\left\|\partial_{x_{0}} u\right\|+\left\|\partial_{x_{1}} u\right\|+\left\|\left(x_{0}-\alpha\left(x^{\prime}\right)\right) \partial_{x_{2}} u\right\|+\|u\| \leq c\left\|^{t} P u\right\|, \\
\forall u \in C_{0}^{\infty}(\overline{\widetilde{\Omega}}): \operatorname{supp} u \subseteq \widetilde{\Omega}_{\bar{x}_{0}, k} .
\end{gathered}
$$




\section{A priori estimates in Sobolev spaces with $s<0$}

First of all, let us obtain a priori estimate in Sobolev spaces with $s<0$ by using the theory of pseudodifferental operators.

Theorem 12 Let us suppose that assumptions (i), (ii) and (iii) hold. For every $\bar{x}_{0} \geq 0$ and for every $s<0$ there exist $k>0$ and $c>0$ such that

$$
\begin{gathered}
\left\|\partial_{x_{0}} u\right\|_{H^{0, s}}+\left\|\partial_{x_{1}} u\right\|_{H^{0, s}}+\left\|\left(x_{0}-\alpha\left(x^{\prime}\right)\right) \partial_{x_{2}} u\right\|_{H^{0, s}}+\|u\|_{H^{0, s}} \leq c\left\|^{t} P u\right\|_{H^{0, s}} \\
\forall u \in C_{0}^{\infty}(\bar{\Omega}): \operatorname{supp} u \subseteq\left[\bar{x}_{0}, \bar{x}_{0}+k\left[\times \Omega_{0}=\Omega_{\bar{x}_{0}, k},\left.D^{\prime} u \cdot n^{\prime}\right|_{S}=0 .\right.\right.
\end{gathered}
$$

Proof Let $u \in C_{0}^{\infty}\left(\overline{\widetilde{\Omega}}_{\bar{x}_{0}, k}\right)$, let $\varphi \in C_{0}^{\infty}(\widetilde{\widetilde{\Omega}})$ such that $\operatorname{supp} \varphi \subseteq \widetilde{\Omega}_{\bar{x}_{0}, k},\left.D^{\prime} \varphi \cdot n^{\prime}\right|_{S}=0$ and $\varphi \equiv 1$ on the support of the projection of $u$ on the plane $x_{0}=\bar{x}_{0}$. Let us set $v_{s}=\varphi\left(x^{\prime}\right) A_{s} u$. Applying the claims of Theorems 4 and 11 if we have $\bar{x}_{0}=0$ or $\bar{x}_{0} \neq 0$, respectively, it results

$$
\left\|\partial_{x_{0}} v_{s}\right\|+\left\|\partial_{x_{1}} v_{s}\right\|+\left\|\left(x_{0}-\alpha\left(x^{\prime}\right)\right) \partial_{x_{2}} v_{s}\right\|+\left\|v_{s}\right\| \leq c\left\|^{t} P v_{s}\right\|
$$

We remark that

$$
\begin{aligned}
& \left\|\partial_{x_{0}} v_{s}\right\|=\left\|\partial_{x_{0}} \varphi\left(x^{\prime}\right) A_{s} u\right\| \\
& \quad=\left\|\varphi\left(x^{\prime}\right) A_{s} \partial_{x_{0}} u\right\| \\
& \quad=\left\|A_{s} \varphi\left(x^{\prime}\right) \partial_{x_{0}} u+\left[\varphi, A_{s}\right] \partial_{x_{0}} u\right\| \\
& \quad \geq\left\|A_{s} \partial_{x_{0}} u\right\|-\left\|R \partial_{x_{0}} u\right\| \\
& \quad \geq\left\|\partial_{x_{0}} u\right\|_{H^{0, s}}-\left\|R \partial_{x_{0}} u\right\|,
\end{aligned}
$$

where $R=\left[\varphi, A_{s}\right] u$ is a regularizing operator with respect to the variable $x^{\prime}$.

On the other hand, making use of Lemma 1 and taking into account that

$$
\begin{gathered}
\partial_{x_{0}}^{2} u=-{ }^{t} P u+\partial_{x_{1}}^{2} u+\left(x_{0}-\alpha\left(x^{\prime}\right)\right)^{2} \partial_{x_{2}}^{2} u+4\left(x_{0}-\alpha\left(x^{\prime}\right)\right) \partial_{x_{2}} \alpha \partial_{x_{2}} u \\
-\frac{1}{i} \sum_{j=0}^{2} a(x) \partial_{x_{j}} u-\frac{1}{i} \sum_{j=0}^{2} \partial_{x_{j}} a(x) u-2\left(\partial_{x_{2}} \alpha\right)^{2} u+b(x) u
\end{gathered}
$$

we obtain

$$
\begin{aligned}
\left\|R \partial_{x_{0}} u\right\| \leq & \left\|R\left(x_{0}-\bar{x}_{0}\right) \partial_{x_{0}}^{2} u\right\| \\
\leq & c\left\|\left(x_{0}-\bar{x}_{0}\right){ }^{t} P u\right\|_{H^{0, s}}+c\left\|\left(x_{0}-\bar{x}_{0}\right) \partial_{x_{0}} u\right\|_{H^{0, s}} \\
& +c\left\|\left(x_{0}-\bar{x}_{0}\right) u\right\|_{H^{0, s}} .
\end{aligned}
$$

Making use of (47) and (48), it follows

$$
\begin{aligned}
& \left\|\partial_{x_{0}} v_{s}\right\| \\
& \quad \geq\left\|\partial_{x_{0}} u\right\|_{H^{0, s}}-c\left\|\left(x_{0}-\bar{x}_{0}\right){ }^{t} P u\right\|_{H^{0, s}}-c\left\|\left(x_{0}-\bar{x}_{0}\right) \partial_{x_{0}} u\right\|_{H^{0, s}}
\end{aligned}
$$




$$
-c\left\|\left(x_{0}-\bar{x}_{0}\right) u\right\|_{H^{0, s}} .
$$

Then choosing $k$ small enough and $\left|x_{0}-\bar{x}_{0}\right|<k$, it results

$$
\left\|\partial_{x_{0}} v_{s}\right\| \geq\left\|\partial_{x_{0}} u\right\|_{H^{0, s}}-c\left\|^{t} P u\right\|_{H^{0, s}} .
$$

Proceeding with the same technique, we easily obtain that

$$
\begin{aligned}
\left\|\partial_{x_{1}} v_{s}\right\| & \geq\left\|\partial_{x_{1}} u\right\|_{H^{0, s}}-c\|u\|_{H^{0, s}} \\
& \geq\left\|\partial_{x_{1}} u\right\|_{H^{0, s}}-c\left\|\left(x_{0}-\bar{x}_{0}\right) \partial_{x_{0}} u\right\|_{H^{0, s}}
\end{aligned}
$$

where we applied Lemma 1. Adding (49) and (50), for $\left|x_{0}-\bar{x}_{0}\right|<k$ with $k$ small enough, we deduce

$$
\left\|\partial_{x_{0}} v_{s}\right\|+\left\|\partial_{x_{1}} v_{s}\right\| \geq\left\|\partial_{x_{0}} u\right\|_{H^{0, s}}+\left\|\partial_{x_{1}} u\right\|_{H^{0, s}}-c\left\|{ }^{t} P u\right\|_{H^{0, s}}
$$

With analogous computations, we have

$$
\left\|\left(x_{0}-\alpha\left(x^{\prime}\right)\right) \partial_{x_{2}} v_{s}\right\| \geq\left\|A_{s}\left(x_{0}-\alpha\left(x^{\prime}\right)\right) \varphi\left(x^{\prime}\right) \partial_{x_{2}} u\right\|-\|R u\|-\left\|B_{s-1} \partial_{x_{1}} u\right\|,
$$

where $B_{s-1}$ is a pseudodifferential operator of order $s-1$. As a consequence, $B_{s-1} \partial_{x_{2}}$ is a pseudodifferential operator of order $s$. By using the continuity property of pseudodifferential operators, it results

$$
\left\|\left(x_{0}-\alpha\left(x^{\prime}\right)\right) \partial_{x_{2}} v_{s}\right\| \geq\left\|\left(x_{0}-\alpha\left(x^{\prime}\right)\right) \partial_{x_{2}} u\right\|_{H^{0, s}}-c\left\|\left(x_{0}-\bar{x}_{0}\right) \partial_{x_{1}} u\right\|_{H^{0, s}}
$$

Adding (51) and (53), for $\left|x_{0}-\bar{x}_{0}\right|<k$ with $k$ small enough, it follows

$$
\begin{aligned}
& \left\|\partial_{x_{0}} v_{s}\right\|+\left\|\partial_{x_{1}} v_{s}\right\|+\left\|\left(x_{0}-\alpha\left(x^{\prime}\right)\right) \partial_{x_{2}} v_{s}\right\| \\
& \quad \geq\left\|\partial_{x_{0}} u\right\|_{H^{0, s}}+\left\|\partial_{x_{1}} u\right\|_{H^{0, s}}+\left\|\left(x_{0}-\alpha\left(x^{\prime}\right)\right) \partial_{x_{2}} u\right\|_{H^{0, s}}-c\left\|^{t} P u\right\|_{H^{0, s}} .
\end{aligned}
$$

Finally we have

$$
\begin{aligned}
\left\|{ }^{t} P v_{s}\right\| & \leq\left\|A_{s}{ }^{t} P u\right\|+\left\|R^{\prime t} P u\right\|+\left\|\varphi\left(x^{\prime}\right)\left[{ }^{t} P, A_{s}\right] u\right\|+\|R u\| \\
& \leq\left\|{ }^{t} P u\right\|_{H^{0, s}}+\left\|\left(x_{0}-\bar{x}_{0}\right) \partial_{x_{0}} u\right\|_{H^{0, s}}+\left\|\varphi\left(x^{\prime}\right)\left[{ }^{t} P, A_{s}\right] u\right\|,
\end{aligned}
$$

where $R^{\prime}$ and $R$ are regularizing operators. Moreover, it results

$$
\varphi\left(x^{\prime}\right)\left[{ }^{t} P, A_{s}\right] u=\varphi\left(x^{\prime}\right)\left[{ }^{t} P_{2}, A_{s}\right] u+\varphi\left(x^{\prime}\right)\left[{ }^{t} P_{1}, A_{s}\right] u+\varphi\left(x^{\prime}\right)\left[{ }^{t} P_{0}, A_{s}\right] u
$$

Evidently, we have

$$
\left[{ }^{t} P_{2}, A_{s}\right] u=B_{s+1} u+B_{s} u
$$


where $B_{s+1}$ and $B_{s}$ are pseudodifferential operators of order $s+1$ and $s$, respectively. The principal symbol of $B_{S+1}$ is

$$
\begin{aligned}
b\left(x, \xi^{\prime}\right)= & -\frac{1}{i}\left(2\left(x_{0}-\alpha\left(x^{\prime}\right)\right)\left(-\partial_{x_{1}} \alpha\left(x^{\prime}\right)\right) \xi_{2}^{2}\right) \varphi\left(x^{\prime}\right) \partial_{\xi_{1}}\left(1+\left|\xi^{\prime}\right|^{2}\right)^{\frac{s}{2}} \\
& -\frac{1}{i}\left(2\left(x_{0}-\alpha\left(x^{\prime}\right)\right)\left(-\partial_{x_{2}} \alpha\left(x^{\prime}\right)\right) \xi_{2}^{2}\right) \varphi\left(x^{\prime}\right) \partial_{\xi_{2}}\left(1+\left|\xi^{\prime}\right|^{2}\right)^{\frac{s}{2}}
\end{aligned}
$$

As a consequence, $B_{s+1} u=\left(x_{0}-\alpha\left(x^{\prime}\right)\right) \partial_{x_{2}} B_{s}^{\prime \prime} u+B_{s}^{\prime} u$, where $B_{s}^{\prime \prime}$ and $B_{s}^{\prime}$ are pseudodifferential operators of order $s$.

Making use of (41), we obtain

$$
\begin{aligned}
\left\|B_{s+1} u\right\| & =\left\|\left(x_{0}-\alpha\left(x^{\prime}\right)\right) \partial_{x_{2}} B_{s}^{\prime \prime} u+B_{s}^{\prime} u\right\| \\
& \leq \varepsilon\left(\left\|^{t} P B_{s}^{\prime \prime} u\right\|+\left\|\partial_{x_{0}} B_{s}^{\prime \prime} u\right\|+\left\|\partial_{x_{1}} B_{s}^{\prime \prime} u\right\|+\left\|B_{s}^{\prime} u\right\|\right)+\left\|B_{s}^{\prime} u\right\| .
\end{aligned}
$$

Then, it follows

$$
\begin{aligned}
& \left\|\varphi\left(x^{\prime}\right)\left[{ }^{t} P_{2}, A_{s}\right] u\right\| \leq \varepsilon\left(\left\|{ }^{t} P B_{s}^{\prime \prime} u\right\|+\left\|\partial_{x_{0}} B_{s}^{\prime \prime} u\right\|\right. \\
& \left.\quad+\left\|\partial_{x_{1}} B_{s}^{\prime \prime} u\right\|+\left\|B_{s}^{\prime \prime} u\right\|\right)+\left\|B_{s} u\right\|+\left\|B_{s}^{\prime} u\right\| \\
& \quad \leq \varepsilon\left(\left\|{ }^{t} P u\right\|_{H^{0, s}}+\left\|\left[{ }^{t} P, B_{s}^{\prime \prime}\right] u\right\|+\left\|\partial_{x_{0}} u\right\|_{H^{0, s}}+\left\|\left[\partial_{x_{0}}, B_{s}^{\prime \prime}\right] u\right\|+\left\|\partial_{x_{1}} u\right\|_{H^{0, s}}\right. \\
& \left.\quad\left\|\left[\partial_{x_{1}}, B_{s}^{\prime \prime}\right] u\right\|+\|u\|_{H^{0, s}}\right)+\left\|B_{s}\left(x_{0}-\bar{x}_{0}\right) \partial_{x_{0}} u\right\|+\left\|B_{s}^{\prime}\left(x_{0}-\bar{x}_{0}\right) \partial_{x_{0}} u\right\| \\
& \quad \leq \varepsilon\left(\left\|{ }^{t} P u\right\|_{H^{0, s}}+\left\|\partial_{x_{0}} u\right\|_{H^{0, s}}+\left\|\partial_{x_{1}} u\right\|_{H^{0, s}}+\|u\|_{H^{0, s}}\right)
\end{aligned}
$$

where we considered $\left|x_{0}-\bar{x}_{0}\right|<k<\varepsilon$.

On the other hand, it results

$$
\varphi\left(x^{\prime}\right)\left[{ }^{t} P_{1}, A_{s}\right] u=B_{s-1} \partial_{x_{0}} u+B_{s} u+B_{s-1} u .
$$

Taking into account Lemma 1, we have

$$
\begin{aligned}
& \left\|\varphi\left(x^{\prime}\right)\left[{ }^{t} P_{1}, A_{s}\right]\right\| \leq\left\|B_{s-1} \partial_{x_{0}} u\right\|+\left\|B_{s} u\right\|+\left\|B_{s-1} u\right\| \\
& \quad \leq c\left\|\left(x_{0}-\bar{x}_{0}\right) B_{s-1} \partial_{x_{0}}^{2} u\right\|+c\|u\|_{H^{0, s}} \\
& \quad \leq c\left\|\left(x_{0}-\bar{x}_{0}\right) B_{s-1} P u\right\|+c\left\|\left(x_{0}-\bar{x}_{0}\right) B_{s} \partial_{x_{1}} u\right\| \\
& \quad+c\left\|\left(x_{0}-\bar{x}_{0}\right) B_{s}^{\prime}\left(x_{0}-\alpha\left(x^{\prime}\right)\right) \partial_{x_{2}} u\right\| \\
& \quad+c\left\|\left(x_{0}-\bar{x}_{0}\right) B_{s}^{\prime \prime} u\right\|+c\left\|\left(x_{0}-\bar{x}_{0}\right) \partial_{x_{0}} u\right\|_{H^{0, s}} \\
& \quad \leq c\left\|\left(x_{0}-\bar{x}_{0}\right) P u\right\|_{H^{0, s}}+c\left\|\left(x_{0}-\bar{x}_{0}\right) \partial_{x_{1}} u\right\|_{H^{0, s}} \\
& \quad+c\left\|\left(x_{0}-\bar{x}_{0}\right)\left(x_{0}-\alpha\left(x^{\prime}\right)\right) \partial_{x_{2}} u\right\|_{H^{0, s}} \\
& \quad+c\left\|\left(x_{0}-\bar{x}_{0}\right) u\right\|_{H^{0, s}}+c\left\|\left(x_{0}-\bar{x}_{0}\right) \partial_{x_{0}} u\right\|_{H^{0, s}} \\
& \quad \leq \varepsilon\left(\|P u\|_{H^{0, s}}+\left\|\partial_{x_{0}} u\right\|_{H^{0, s}}+\left\|\partial_{x_{1}} u\right\|_{H^{0, s}}\right. \\
& \left.\quad+\left\|\left(x_{0}-\alpha\left(x^{\prime}\right)\right) \partial_{x_{2}} u\right\|_{H^{0, s}}+\|u\|_{H^{0, s}}\right)
\end{aligned}
$$

where $B_{s}, B_{s}^{\prime}, B_{s}^{\prime \prime}$ are pseudodifferential operators of order $s, B_{s-1}$ is a pseudodifferential operator of order $s-1$ and we supposed that $0<\left|x_{0}-\bar{x}_{0}\right|<\frac{\varepsilon}{c}$. 
It is easy to obtain

$$
\begin{aligned}
\left\|\varphi\left(x^{\prime}\right)\left[{ }^{t} P_{0}, A_{s}\right] u\right\| & \leq c\|u\|_{H^{0, s}} \\
& \leq c\left\|\left(x_{0}-\bar{x}_{0}\right) \partial_{x_{0}} u\right\|_{H^{0, s}} \\
& \leq \varepsilon\left\|\partial_{x_{0}} u\right\|_{H^{0, s}}^{2} .
\end{aligned}
$$

By using (57), (58), (59), it follows

$$
\begin{aligned}
& \left\|\varphi\left(x^{\prime}\right)\left[{ }^{t} P, A_{s}\right] u\right\| \\
& \quad \leq \varepsilon\left(\left\|{ }^{t} P u\right\|_{H^{0, s}}+\left\|\partial_{x_{0}} u\right\|_{H^{0, s}}+\left\|\partial_{x_{1}} u\right\|_{H^{0, s}}+\left\|\left(x_{0}-\alpha\left(x^{\prime}\right)\right) \partial_{x_{2}} u\right\|_{H^{0, s}}+\|u\|_{H^{0, s}}\right) .
\end{aligned}
$$

Making use of (46), (54) and the previous estimate, the claim is achieved.

With analogous techniques used to prove Theorem 12 but making use of Theorems 3 and 10 instead of Theorems 4 and 11, respectively, we can establish the following relevant estimate.

Theorem 13 Let us suppose that assumptions (i), (ii) and (iii) hold. For every $\bar{x}_{0} \geq 0$ and for every $s<0$ there exist $k>0$ and $c>0$ such that

$$
\begin{gathered}
\left\|\partial_{x_{0}} u\right\|_{H^{0, s}}+\left\|\partial_{x_{1}} u\right\|_{H^{0, s}}+\left\|\left(x_{0}-\alpha\left(x^{\prime}\right)\right) \partial_{x_{2}} u\right\|_{H^{0, s}}+\|u\|_{H^{0, s}} \leq c\left\|^{t} P u\right\|_{H^{0, s}}, \\
\forall u \in C_{0}^{\infty}(\bar{\Omega}): \operatorname{supp} u \subseteq\left[\bar{x}_{0}, \bar{x}_{0}+k\left[\times \Omega_{0}=\Omega_{\bar{x}_{0}, k},\left.L^{\prime} u \cdot n^{\prime}\right|_{S}=0 .\right.\right.
\end{gathered}
$$

\section{Global estimates}

Now, we obtain a global estimate very useful in order to prove the existence of a solution to the Cauchy-Neumann problem (1).

Theorem 14 Let us suppose that assumptions (i), (ii) and (iii) hold. For every $h>0$ and $s \leq 0$ there exists $c>0$ such that

$$
\begin{gathered}
\left\|\partial_{x_{0}} u\right\|_{H^{0, s}}+\left\|\partial_{x_{1}} u\right\|_{H^{0, s}}+\left\|\left(x_{0}-\alpha\left(x^{\prime}\right)\right) \partial_{x_{2}} u\right\|_{H^{0, s}}+\|u\|_{H^{0, s}} \leq c\left\|^{t} P u\right\|_{H^{0,-s}}, \\
\forall u \in C_{0}^{\infty}(\bar{\Omega}): \operatorname{supp} u \subseteq \Omega_{h},\left.D^{\prime} u \cdot n^{\prime}\right|_{S}=0 .
\end{gathered}
$$

Proof Let $h>0$, setting $\Omega_{h}=\left[0, h\left[\times \Omega_{0}\right.\right.$, for the compactness of $[0, h] \times \bar{\Omega}_{0}$, there exists a finite number of subsets $\left\{\Omega_{1}, \Omega_{2}, \ldots, \Omega_{p}\right\}$ of $\Omega_{h}$, given by

$$
\Omega_{1}=\left[0, k_{1}\left[\times \Omega_{0}, \Omega_{2}=\left[k_{1}^{\prime}, k_{2}\left[\times \Omega_{0}, \ldots, \Omega_{p}=\left[k_{p-1}^{\prime}, k_{p}\left[\times \Omega_{0},\right.\right.\right.\right.\right.\right.
$$

with $k_{0}=0, k_{p}=h, k_{i-1}<k_{i}^{\prime}<k_{i}$, for every $i=1, \ldots, p$, and such that (45) holds in every $\Omega_{i}$, for $i=1, \ldots, p$.

Let $u \in C_{0}^{\infty}\left(\Omega_{h}\right)$, with $\left.D^{\prime} u \cdot n^{\prime}\right|_{S}=0$, let $\varphi \in C_{0}^{\infty}\left(\left[0, k_{1}[)\right.\right.$, with $\varphi \equiv 1$ on $\left[0, k_{1}^{\prime}[\right.$ and $0 \leq \varphi \leq 1$ in $\left[0, k_{1}[\right.$. Rewriting (45) for $\varphi u$, it results

$$
\left\|\partial_{x_{0}} \varphi u\right\|_{H^{0, s}}+\left\|\partial_{x_{1}} \varphi u\right\|_{H^{0, s}}+\left\|\left(x_{0}-\alpha\left(x^{\prime}\right)\right) \partial_{x_{2}} \varphi u\right\|_{H^{0, s}}+\|\varphi u\|_{H^{0, s}}
$$




$$
\begin{aligned}
& \leq c\|P \varphi u\|_{H^{0, s}} \\
& \leq c\|P u\|_{H^{0, s}}+c\|[P, \varphi] u\|_{H^{0, s}} \\
& \leq c\|P u\|_{H^{0, s}}+c\left\|\partial_{x_{0}} \varphi \partial_{x_{0}} u\right\|_{H^{0, s}}+c\left\|\left(\partial_{x_{0}}^{2} \varphi\right) u\right\|_{H^{0, s}} \\
& \leq c\|P u\|_{H^{0, s}}+c\left\|\partial_{x_{0}} u\right\|_{H^{0, s}\left(\left[k_{1}^{\prime}, k_{1}\left[\times \Omega_{0}\right)\right.\right.}+c\|u\|_{H^{0, s}\left(\left[k_{1}^{\prime}, k_{1}\left[\times \Omega_{0}\right)\right.\right.} \\
& \leq c\|P u\|_{H^{0, s}}+c\left\|\partial_{x_{0}} u\right\|_{H^{0, s}\left(\left[k_{1}^{\prime}, k_{2}^{\prime}\left[\times \Omega_{0}\right)\right.\right.}+c\|u\|_{H^{0, s}\left(\left[k_{1}^{\prime}, k_{2}^{\prime}\left[\times \Omega_{0}\right)\right.\right.} \\
& \leq c\|P u\|_{H^{0, s}}+c\left\|\partial_{x_{0}} \varphi_{1} u\right\|_{H^{0, s}\left(\left[k_{1}^{\prime}, k_{2}\left[\times \Omega_{0}\right)\right.\right.}+c\left\|\varphi_{1} u\right\|_{H^{0, s}\left(\left[k_{1}^{\prime}, k_{2}\left[\times \Omega_{0}\right)\right.\right.},
\end{aligned}
$$

where $\varphi_{1} \in C_{0}^{\infty}\left(\Omega_{0}\right)$ such that $\operatorname{supp} \varphi_{1} \subseteq\left[k_{1}^{\prime}, k_{2}\left[, \varphi_{1} \equiv 1\right.\right.$ in $\left[k_{1}^{\prime}, k_{2}^{\prime}\right] \times \Omega_{0}$.

We can deduce that

$$
\begin{aligned}
& \left\|\partial_{x_{0}} \varphi_{i-1} u\right\|_{H^{0, s}}+\left\|\partial_{x_{1}} \varphi_{i-1} u\right\|_{H^{0, s}}+\left\|\left(x_{0}-\alpha\left(x^{\prime}\right)\right) \partial_{x_{2}} \varphi_{i-1} u\right\|_{H^{0, s}}+\left\|\varphi_{i-1} u\right\|_{H^{0, s}} \\
& \quad \leq c\|P u\|_{H^{0, s}}+c\left\|\partial_{x_{0}} \varphi_{i} u\right\|_{H^{0, s}\left(\left[k_{i}^{\prime}, k_{i+1}\left[\times \Omega_{0}\right)\right.\right.}+c\left\|\varphi_{i} u\right\|_{H^{0, s}\left(\left[k_{i}^{\prime}, k_{i+1}\left[\times \Omega_{0}\right)\right.\right.},
\end{aligned}
$$

where $\varphi_{0}=\varphi$ and $\varphi_{i} \in C_{0}^{\infty}\left(\left[0, h[)\right.\right.$ such that $\operatorname{supp} \varphi_{i} \subseteq\left[k_{i}^{\prime}, k_{i+1}[\right.$, for every $i=$ $1, \ldots, p$.

On the other hand, we have

$$
\begin{aligned}
& \left\|\partial_{x_{0}} \varphi_{p-1} u\right\|_{H^{0, s}}+\left\|\partial_{x_{1}} \varphi_{p-1} u\right\|_{H^{0, s}}+\left\|\left(x_{0}-\alpha\left(x^{\prime}\right)\right) \partial_{x_{2}} \varphi_{p-1} u\right\|_{H^{0, s}}+\left\|\varphi_{p-1} u\right\|_{H^{0, s}} \\
& \quad \leq c\|P u\|_{H^{0, s}}+c\left\|\partial_{x_{0}} \varphi_{p} u\right\|_{H^{0, s}\left(\Omega_{p}\right)}+c\left\|\varphi_{p} u\right\|_{H^{0, s}\left(\Omega_{p}\right)} \\
& \quad \leq c\|P u\|_{H^{0, s}}+c\left(\left\|\partial_{x_{0}} u\right\|_{H^{0, s}\left(\Omega_{p}\right)}+\|u\|_{H^{0, s}\left(\Omega_{p}\right)}\right) \\
& \quad \leq c\|P u\|_{H^{0, s}} .
\end{aligned}
$$

By using (47), (60) and proceeding by recurrence on $i$, we easily obtain

$$
\begin{aligned}
& \left\|\partial_{x_{0}} \varphi_{i} u\right\|_{H^{0, s}}+\left\|\partial_{x_{1}} \varphi_{i} u\right\|_{H^{0, s}}+\left\|\left(x_{0}-\alpha\left(x^{\prime}\right)\right) \partial_{x_{2}} \varphi_{i} u\right\|_{H^{0, s}} \\
& \quad+\left\|\varphi_{i} u\right\|_{H^{0, s}} \leq c\|P u\|_{H^{0, s}},
\end{aligned}
$$

for $i=1, \ldots, p$. Taking into account the previous inequality, we have

$$
\begin{gathered}
\left\|\partial_{x_{0}} u\right\|_{H^{0, s}}+\left\|\partial_{x_{1}} u\right\|_{H^{0, s}}+\left\|\left(x_{0}-\alpha\left(x^{\prime}\right)\right) \partial_{x_{2}} u\right\|_{H^{0, s}}+\|u\|_{H^{0, s}} \leq c\|P u\|_{H^{0, s}} \\
\forall u \in C_{0}^{\infty}(\bar{\Omega}): \operatorname{supp} u \subseteq \Omega_{h},\left.D^{\prime} u \cdot n^{\prime}\right|_{S}=0
\end{gathered}
$$

For the arbitrariness of $h,(61)$ holds for every $u \in C_{0}^{\infty}(\bar{\Omega})$ such that $\left.D^{\prime} u \cdot n^{\prime}\right|_{S}=0$. The proof is thereby completed.

Proceeding analogously as in the proof of Theorem 14 but by using Theorems 3 and 10 instead of Theorems 2 and 7, respectively, we obtain a global estimate for the Cauchy-Robin problem (2).

Theorem 15 Let us suppose that assumptions (i), (ii) and (iii) hold. For every $h>0$ and $s \leq 0$ there exists $c>0$ such that

$$
\left\|\partial_{x_{0}} u\right\|_{H^{0, s}}+\left\|\partial_{x_{1}} u\right\|_{H^{0, s}}+\left\|\left(x_{0}-\alpha\left(x^{\prime}\right)\right) \partial_{x_{2}} u\right\|_{H^{0, s}}+\|u\|_{H^{0, s}} \leq c\left\|^{t} P u\right\|_{H^{0,-s}},
$$




$$
\forall u \in C_{0}^{\infty}(\bar{\Omega}): \operatorname{supp} u \subseteq \Omega_{h},\left.L^{\prime} u \cdot n^{\prime}\right|_{S}=0
$$

\section{Existence and regularity results}

This section is devoted to establish existence and regularity results for the CauchyNeumann problem (1) and the Cauchy-Robin problem (2).

Theorem 16 Let $f \in H_{l o c}^{r}(\bar{\Omega})$, with $r \geq 2$. Then, for every $h>0$ there exists $v \in H^{0, s}\left(\Omega_{h}\right)$, with $0 \leq s \leq r$ such that

$$
\left(v,{ }^{t} P u\right)=(f, u), \quad \forall u \in C_{0}^{\infty}(\bar{\Omega}): \operatorname{supp} u \subseteq \Omega_{h},\left.D^{\prime} u \cdot n^{\prime}\right|_{S}=0 .
$$

Proof Let $B^{\prime}$ be the subspace of distributions of $H^{0,-s}\left(\Omega_{h}\right)$ defined on test functions $\varphi \in C_{0}^{\infty}\left(\left[0, h\left[\times \bar{\Omega}_{0}\right)\right.\right.$ such that $\left.D^{\prime} \varphi \cdot u^{\prime}\right|_{S}=0$. Let $B$ be contained in $B^{\prime}$. Let us define a linear continuous functional in $B$, as follows

$$
F(\psi)=F\left({ }^{t} P u\right)=(f, u), \quad \forall \psi \in B
$$

Taking into account Theorem 12, it results

$$
|F(\psi)|=|(f, u)| \leq\|f\|_{H^{0, s}}\|u\|_{H^{0,-s}} \leq c\|f\|_{H^{0, s}}\left\|^{t} P u\right\|_{H^{0,-s}} \leq c^{\prime}\|\psi\|_{H^{0,-s}} .
$$

As a consequence, we can extend $F$ in $H^{0,-s}\left(\Omega_{h}\right) \cap B^{\prime}$. Making use of a representation theorem, there exists $v \in H^{0, s}\left(\Omega_{h}\right) \cap B^{*}$ such that

$$
F(w)=(v, w), \quad \forall w \in H^{0,-s}\left(\Omega_{h}\right)
$$

Then, it results

$F(\psi)=(v, \psi)=\left(v,{ }^{t} P u\right)=(f, u), \quad \forall u \in C_{0}^{\infty}(\bar{\Omega}): \operatorname{supp} u \subseteq \Omega_{h},\left.D^{\prime} u \cdot n^{\prime}\right|_{S}=0$

We proved that for every $h>0$ there exists $v \in H^{0, s}\left(\Omega_{h}\right)$, with $0 \leq s \leq r$, such that

$$
P v=f, \quad \text { in the sense of distributions. }
$$

Hence, $v$ verifies the following equality:

$$
\partial_{x_{0}}^{2} v+a_{0}(x) \partial_{x_{0}} v+b(x) v=f+\left(P-\partial_{x_{0}}^{2}-a_{0}(x) \partial_{x_{0}}-b(x)\right) v
$$

Since $f+\left(P-\partial_{x_{0}}^{2}-a_{0}(x) \partial_{x_{0}}-b(x)\right) v \in H^{0, r-2}\left(\Omega_{h}\right)$, we have $v \in H^{2, r-2}\left(\Omega_{h}\right)$. Therefore, proceeding by induction, we deduce

$$
v \in H^{s, r-s}\left(\Omega_{h}\right), \quad \forall s \leq r .
$$


As a consequence, it follows

$$
v \in H^{r}\left(\Omega_{h}\right) .
$$

Then, there exists $v \in H^{r}\left(\Omega_{h}\right)$, with $r \geq 2$, such that

$$
\left(v,{ }^{t} P u\right)=(f, u), \quad \forall u \in C_{0}^{\infty}\left(\Omega_{h}\right):\left.D^{\prime} u \cdot n^{\prime}\right|_{S}=0 .
$$

Taking into account (62) and integrating by parts, we obtain

$$
(P v, u)=(f, u), \quad \forall u \in C_{0}^{\infty}\left(\text { int } \Omega_{h}\right)
$$

Hence, it results

$$
P v=f, \quad \text { a.e. in } \Omega_{h} .
$$

Integrating again by parts in (62), for every $u \in C_{0}^{\infty}\left(\Omega_{h}\right)$ such that $\left.u\right|_{\Omega_{0}}=0$, $\left.\partial_{x_{0}} u\right|_{\Omega_{0}}=0,\left.D^{\prime} u \cdot n^{\prime}\right|_{S}=0$, we have

$$
\begin{aligned}
& (P v, u)-\int_{S}\left(\partial_{x_{1}} v \cdot n_{1}+\left(x_{0}-\alpha\left(x^{\prime}\right)\right)^{2} \partial_{x_{2}} v \cdot n_{2}-a_{1}(x) n_{1} v-a_{2}(x) n_{2} v\right) u d \sigma \\
& \quad=(f, u)
\end{aligned}
$$

which implies

$$
\int_{S}\left(L^{\prime} v \cdot n^{\prime}\right) u d \sigma=0
$$

Then, it follows

$$
\left.L^{\prime} v \cdot n^{\prime}\right|_{S}=0
$$

Finally, integrating again by parts in (62), for every $u \in C_{0}^{\infty}\left(\Omega_{h}\right)$ such that $\left.u\right|_{S}=0$, $\left.\partial_{x_{i}} u\right|_{S}=0$, with $i=1,2$, and supposing that either $\left.\partial_{x_{0}} u\right|_{\Omega_{0}}=0$ or $\left.u\right|_{\Omega_{0}}=0$, we get

$$
(P v, u)-\int_{\Omega_{0}} v \partial_{x_{0}} u d x_{1} d x_{2}=(f, u) .
$$

As a consequence, it results

$$
\left.v\right|_{\Omega_{0}}=0 .
$$

Moreover, we have

$$
(P v, u)-\int_{\Omega_{0}} \partial_{x_{0}} v \cdot u d x_{1} d x_{2}=(f, u) .
$$


Hence, we obtain

$$
\left.\partial_{x_{0}} v\right|_{\Omega_{0}}=0
$$

Making use of (63), (64), (65) and (66), it follows that there exists $v \in H^{r}\left(\Omega_{h}\right)$ such that

$$
\left\{\begin{array}{l}
P v=f, \quad \text { in } \Omega_{h}, \\
\left.v\right|_{\Omega_{0}}=0,\left.\quad \frac{d v}{d n}\right|_{\Omega_{0}}=0,\left.L^{\prime} v \cdot n^{\prime}\right|_{S}=0 .
\end{array}\right.
$$

Instead if $B$ is the space of functions $\psi={ }^{t} P u$, with $u \in C_{0}^{\infty}(\bar{\Omega})$ such that supp $u \subseteq$ $\bar{\Omega}_{h}$ and $\left.L^{\prime} u \cdot n^{\prime}\right|_{S}=0$, proceeding as done before, we obtain the claim.

Moreover, with analogous proof of Theorem 16 but applying Theorem 13 instead of Theorem 12 and considering as $B^{\prime}$ the subspace of distributions of $H^{0,-s}\left(\Omega_{h}\right)$ defined on test functions $\varphi \in C^{\infty}\left(\left[0, h\left[\times \bar{\Omega}_{0}\right)\right.\right.$ such that $\left.L^{\prime} \varphi \cdot n^{\prime}\right|_{S}=0$, the following results holds.

Theorem 17 Let $f \in H_{l o c}^{r}(\bar{\Omega})$, with $r \geq 2$. Then, for every $h>0$ there exists $v \in H^{0, s}\left(\Omega_{h}\right)$, with $0 \leq s \leq r$ such that

$$
\left(v,{ }^{t} P u\right)=(f, u), \quad \forall u \in C_{0}^{\infty}(\bar{\Omega}): \operatorname{supp} u \subseteq \Omega_{h},\left.L^{\prime} u \cdot n^{\prime}\right|_{S}=0 .
$$

Integrating by parts (67), as done before, it follows that there exists $v \in H^{r}\left(\Omega_{h}\right)$ such that

$$
\left\{\begin{array}{l}
P v=f, \quad \text { in } \Omega_{h}, \\
\left.v\right|_{\Omega_{0}}=0,\left.\quad \frac{d v}{d n}\right|_{\Omega_{0}}=0,\left.\quad D^{\prime} v \cdot n^{\prime}\right|_{S}=0 .
\end{array}\right.
$$

with $f \in H_{l o c}^{r}(\bar{\Omega})$.

\section{Uniqueness of the solution}

In order to establish the uniqueness of a solution to the problem (1), we prove, as a first step, the existence of a solution to the following problems

$$
\left\{\begin{array}{l}
\left.{ }^{t} P w=f, \quad \text { in } \Omega_{h}=\right] 0, h\left[\times \Omega_{0}\right. \\
w\left(h, x^{\prime}\right)=0, \partial_{x_{0}} w\left(h, x^{\prime}\right)=0,\left.D^{\prime} w \cdot n^{\prime}\right|_{S}=0
\end{array}\right.
$$

and

$$
\left\{\begin{array}{l}
\left.{ }^{t} P w=f, \quad \text { in } \Omega_{h}=\right] 0, h\left[\times \Omega_{0}\right. \\
w\left(h, x^{\prime}\right)=0, \quad \partial_{x_{0}} w\left(h, x^{\prime}\right)=0,\left.L^{\prime} w \cdot n^{\prime}\right|_{S}=0
\end{array}\right.
$$


with $f \in H^{r}\left(\Omega_{h}\right)$. To this aim, we can proceed in analogous way as done in the proofs of the theorems in Sects. 4, 5, 6, 7 considering, for every $\left.\bar{x}_{0} \in\right] 0, h[$,

$$
\begin{aligned}
\Omega_{\bar{x}_{0}, k} & =\left\{x \in \bar{\Omega}: x_{0} \in\right] \bar{x}_{0}-k, \bar{x}_{0}\left[\times \Omega_{0}\right\}, \\
\Omega_{\bar{x}_{0}, k, \eta} & =\left\{x \in \Omega_{\bar{x}_{0}, k}:\left(\frac{1}{5}+\eta\right) \alpha\left(x^{\prime}\right)+\left(\frac{4}{5}-\eta\right) \bar{x}_{0} \leq x_{0} \leq \eta \bar{x}_{0}+(1-\eta) \alpha\left(x^{\prime}\right)\right\}, \\
\Omega_{\bar{x}_{0}, k, \eta}^{\prime} & =\left\{x \in \Omega_{\bar{x}_{0}, k, \eta}:\left|\partial_{x_{1}} \alpha\left(x^{\prime}\right)\right| \geq 1\right\}, \\
\Omega_{\bar{x}_{0}, k, \eta, \eta^{\prime}}^{\prime} & =\left\{x \in \Omega_{\bar{x}_{0}, k, \eta}:\left|\partial_{x_{1}} \alpha\left(x^{\prime}\right)\right| \geq 1-\eta^{\prime}\right\},
\end{aligned}
$$

where $\frac{4}{5}<\eta<1$ and $0<k<h$, and the operator ${ }^{t} P$ instead of the operator $P$. With these modifications and under assumptions (i), (ii) and (iii), we obtain that there exist solutions to problems (68) and (69), with $f \in H_{l o c}^{r}\left(\Omega_{0}\right)$. As a consequence, there exists a solution $w \in C^{\infty}\left(\Omega_{h}\right)$ to the problem

$$
\left\{\begin{array}{l}
{ }^{t} P w=0, \quad \text { in } \Omega_{h}, \\
w\left(h, x^{\prime}\right)=0, \partial_{x_{0}} w\left(h, x^{\prime}\right)=\varphi\left(x^{\prime}\right),\left.D^{\prime} w \cdot n^{\prime}\right|_{S}=0
\end{array}\right.
$$

and exists a solution $w \in C^{\infty}\left(\Omega_{h}\right)$ to the problem

$$
\left\{\begin{array}{l}
{ }^{t} P w=0, \quad \text { in } \Omega_{h}, \\
w\left(h, x^{\prime}\right)=0, \partial_{x_{0}} w\left(h, x^{\prime}\right)=\varphi\left(x^{\prime}\right),\left.L^{\prime} w \cdot n^{\prime}\right|_{S}=0
\end{array}\right.
$$

with $\varphi \in C_{0}^{\infty}\left(\Omega_{0}\right)$.

Now, if $v \in H_{l o c}^{r}\left(\Omega_{h^{\prime}}\right)$, with $r \geq 2$, is a solution to the problem

$$
\left\{\begin{array}{l}
\left.P v=0, \quad \text { in } \Omega_{h^{\prime}}=\right] 0, h^{\prime}\left[\times \Omega_{0}, \text { with } h^{\prime} \geq h\right. \\
v\left(0, x^{\prime}\right)=0, \partial_{x_{0}} v\left(0, x^{\prime}\right)=0,\left.L^{\prime} v \cdot n^{\prime}\right|_{S}=0
\end{array}\right.
$$

and $w$ is a solution to (70), it results

$$
0=\left(v,{ }^{t} P w\right)=(P v, w)+\int_{\Omega_{0}} v\left(h, x^{\prime}\right) \varphi\left(x^{\prime}\right) d x^{\prime}=\int_{\Omega_{0}} v\left(h, x^{\prime}\right) \varphi\left(x^{\prime}\right) d x^{\prime}
$$

For the arbitrary of $\varphi$, it follows that $v\left(h, x^{\prime}\right)=0$. Hence, we get that $v=0$ in $\left.\Omega_{h^{\prime}}=\right] 0, h^{\prime}\left[\times \Omega_{0}\right.$. Moreover, for the arbitrary of $h^{\prime}$, it results that, under assumptions (i) and (ii), the problem

$$
\left\{\begin{array}{l}
P v=f, \quad \text { in } \Omega=] 0,+\infty\left[\times \Omega_{0}\right. \\
\left.v\right|_{\Omega_{0}}=0,\left.\quad \partial_{x_{0}} v\right|_{\Omega_{0}}=0,\left.L^{\prime} v \cdot n^{\prime}\right|_{S}=0
\end{array}\right.
$$


with $f \in H_{l o c}^{2}(\bar{\Omega})$, admits a unique solution $v \in H_{l o c}^{r}(\bar{\Omega})$, with $r \geq 2$. Instead, let $v \in H_{l o c}^{r}(\bar{\Omega})$ be a solution to the problem

$$
\left\{\begin{array}{l}
P v=0, \quad \text { in } \Omega=] 0, h^{\prime}\left[\times \Omega_{0}, \text { with } h^{\prime} \geq h\right. \\
v\left(0, x^{\prime}\right)=0, \partial_{x_{0}} v\left(0, x^{\prime}\right)=0,\left.D^{\prime} v \cdot n^{\prime}\right|_{S}=0
\end{array}\right.
$$

and $w$ is a solution to (71), it follows

$$
0=\left(v,{ }^{t} P w\right)=(P v, w)+\int_{\Omega_{0}} v\left(h, x^{\prime}\right) \varphi\left(x^{\prime}\right) d x^{\prime}=\int_{\Omega_{0}} v\left(h, x^{\prime}\right) \varphi\left(x^{\prime}\right) d x^{\prime} .
$$

Therefore, under assumptions (i), (ii) and (iii), also the problem

$$
\left\{\begin{array}{l}
P v=f, \quad \text { in } \Omega=] 0,+\infty\left[\times \Omega_{0},\right. \\
\left.v\right|_{\Omega_{0}}=0,\left.\quad \partial_{x_{0}} v\right|_{\Omega_{0}}=0,\left.D^{\prime} v \cdot n^{\prime}\right|_{S}=0
\end{array}\right.
$$

with $f \in H_{l o c}^{r}(\bar{\Omega})$, admits a unique solution.

Funding Open access funding provided by Università degli Studi di Napoli Federico II within the CRUICARE Agreement.

Open Access This article is licensed under a Creative Commons Attribution 4.0 International License, which permits use, sharing, adaptation, distribution and reproduction in any medium or format, as long as you give appropriate credit to the original author(s) and the source, provide a link to the Creative Commons licence, and indicate if changes were made. The images or other third party material in this article are included in the article's Creative Commons licence, unless indicated otherwise in a credit line to the material. If material is not included in the article's Creative Commons licence and your intended use is not permitted by statutory regulation or exceeds the permitted use, you will need to obtain permission directly from the copyright holder. To view a copy of this licence, visit http://creativecommons.org/licenses/by/4.0/.

\section{References}

1. Barbagallo, A., Esposito, V.: A priori estimate for a class of hyperbolic equations with double characteristics. Rend. Acc. Sc. Fis. Mat. Napoli LXXXI, 113-120 (2014)

2. Barbagallo, A., Esposito, V.: A global existence and uniqueness result for a class of hyperbolic operators. Ricerche Mat. 63, 25-40 (2014)

3. Barbagallo, A., Esposito, V.: Energy estimates for the Cauchy problem associated to a class of hyperbolic operators with double characteristics in presence of transition. Ricerche Mat. 64, 243-249 (2015)

4. Barbagallo, A., Esposito, V.: The Cauchy-Dirichlet problem for a class of hyperbolic operators with double characteristics in presence of transition. J. Math. Anal. Appl. 442, 149-170 (2016)

5. Barbagallo, A., Esposito, V.: On hyperbolic equations with double characteristics in the presence of transition. Bound. Value Probl. Art. no. 152 (2016)

6. Barbagallo, A., Esposito, V.: A priori estimates in Sobolev spaces for a class of hyperbolic operators in presence of transition. J. Hyperb. Differ. Equ. 16, 245-270 (2019)

7. Bernardi, E., Bove, A., Parenti, C.: Geometric results for a class of hyperbolic operators with double characteristics. J. Funct. Anal. 116, 62-82 (1993)

8. Bernardi, E., Parenti, C., Parmeggiani, A.: The Cauchy problem for hyperbolic operators with double characteristics in presence of transition. Commun. Part. Differ. Equ. 37, 1315-1356 (2012)

9. Hörmander, L.: The Cauchy problem for differential equations with double characteristics. J. Anal. Math. 32, 118-196 (1977) 
10. Ivrii, V.J., Petkov, V.M.: Necessary conditions for well-posedness of the Cauchy problem for nonstrictly hyperbolic equations. Russ. Math. Surv. 29, 1-70 (1974)

Publisher's Note Springer Nature remains neutral with regard to jurisdictional claims in published maps and institutional affiliations. 